\title{
Boundary effects on the nonequilibrium structure factor of fluids below the Rayleigh-Bénard instability
}

\author{
José M. Ortiz de Zárate* \\ Departamento de Física Aplicada I, Facultad de Ciencias Físicas, Universidad Complutense, 28040 Madrid, Spain \\ Jan V. Sengers \\ Institute for Physical Science and Technology and Department of Chemical Engineering, University of Maryland, \\ College Park, Maryland 20742
}

(Received 26 December 2001; revised manuscript received 1 May 2002; published 18 September 2002)

\begin{abstract}
We consider a horizontal fluid layer between two rigid boundaries, maintained in a stationary thermal nonequilibrium state below the convective Rayleigh-Bénard instability. We derive an explicit expression for the nonequilibrium structure factor in a first-order Galerkin approximation valid for negative and positive Rayleigh numbers $R$ up to the critical Rayleigh number $R_{c}$ associated with the appearance of convection. The results obtained for rigid boundaries by the Galerkin-approximation method are compared with exact results previously derived for the case of free boundaries. The nonequilibrium structure factor exhibits a maximum as a function of the wave number $q$ of the fluctuations. This maximum is associated with a crossover from a $q^{-4}$ dependence for larger $q$ to a $q^{2}$ dependence for small $q$. This maximum is present at both negative and positive $R$, becomes pronounced at positive $R$ and diverges as $R$ approaches the critical value $R_{c}$.
\end{abstract}

DOI: 10.1103/PhysRevE.66.036305 PACS number(s): 47.20.-k, 05.40.-a, 05.70.Ln, 44.25.+f

\section{INTRODUCTION}

During the past years considerable effort has been devoted to the study of hydrodynamic fluctuations in liquids in stationary thermal nonequilibrium states, particularly when a liquid layer is subjected to a constant temperature gradient, $\boldsymbol{\nabla} T_{0}$. It turns out that density or temperature fluctuations in such nonequilibrium states become spatially long ranged, even in the absence of any convective instabilities [1].

The long-range nature of the fluctuations manifests itself as a wave-number-dependent anisotropic enhancement in the Rayleigh component $S(q)$ of the structure factor. The first correct calculation of the nonequilibrium structure factor of a fluid, without taking into account gravity or finite-size effects, was performed by Kirkpatrick et al. using modecoupling theory [2] and later confirmed by fluctuating hydrodynamics [3-6]. The main conclusion is that the nonequilibrium enhancement of $S(q)$ is maximum for fluctuations with wave vector $\mathbf{q} \perp \boldsymbol{\nabla} T_{0}$, in which case it varies as $q^{-4}$ with the wave number $q$ of the fluctuations. The divergence of the structure factor for small $q$ as $q^{-4}$ cannot go on indefinitely up to wave numbers corresponding to macroscopic wavelengths. Specifically, two sources can be identified that will cause deviations from the $q^{-4}$ behavior at very small wave numbers: gravity and finite-size effects. Gravity causes the $q^{-4}$ divergence to be quenched, the structure factor reaching a constant limit in $q \rightarrow 0$, as was elucidated by Segrè et al. $[7,8]$. They found that the static structure factor of a fluid subjected to a stationary temperature gradient $\nabla T_{0}$ in the presence of gravity can be written as

$$
S(q)=S_{E}\left[1+\frac{\widetilde{S}_{\mathrm{NE}}^{0}}{\sigma+1} \frac{1}{q^{4} L^{4}-R}\right],
$$

\footnotetext{
*Author to whom correspondence should be addressed. Email address: fiap102@sis.ucm.es
}

where $S_{E}$ is the well-known isotropic expression for the intensity of the fluctuations in thermodynamic equilibrium,

$$
S_{E}=\rho^{2} \varkappa_{T} k_{B} T \frac{\gamma-1}{\gamma},
$$

while $\widetilde{S}_{\mathrm{NE}}^{0}$ represents the strength of the nonequilibrium enhancement of the structure factor, and is given by

$$
\widetilde{S}_{\mathrm{NE}}^{0}=\sigma R+\frac{\left(c_{P} / T\right) L^{4}}{D_{T}^{2}}\left(\nabla T_{0}\right)^{2} .
$$

In the equations above, $\sigma$ denotes the Prandtl number, $R$ the Rayleigh number, $\rho$ the density, $\varkappa_{T}$ the isothermal compressibility, $T$ the average temperature, $\gamma$ the heat-capacity ratio, $c_{P}$ the isobaric specific heat capacity, and $D_{T}$ the thermal diffusivity. In Eqs. (1) and (3) we have also introduced the finite height $L$ of the horizontal fluid layer so as to elucidate the connection of $S(q)$ with the Rayleigh number $R$ and to facilitate a comparison with the results to be presented in this paper where finite-size effects will be considered. However, Eq. (1) represents the bulk structure factor of the fluid without any finite-size effects and one can readily verify that it does not depend explicitly upon the finite height $L$.

We note that $S(q)$, as given by Eq. (1), contains an equilibrium contribution and a nonequilibrium enhancement. The equilibrium contribution $S_{E}$ is independent of the wave number $q$ and equals the traditional formula for the isotropic Rayleigh-scattering intensity [9]. The nonequilibrium enhancement is proportional to the square of the temperature gradient through $\widetilde{S}_{\mathrm{NE}}^{0}$. For $q^{4} \gg R / L^{4}, S(q)$ varies as $q^{-4}$, in accordance with the asymptotic behavior first found by Kirkpatrick et al. [2]. This dependence of the nonequilibrium contribution to the structure factor on $q^{-4}$ has been experimentally verified by several small-angle Rayleigh-scattering 
experiments [10-13]. For $q \rightarrow 0$, the nonequilibrium structure factor reaches a constant value, which is a consequence of the presence of gravity. The gravitationally induced saturation of the $q^{-4}$ divergence of the nonequilibrium structure factor has been confirmed by Vailati and Giglio $[14,15]$ from ultra-low-angle light-scattering experiments.

As investigated by several authors [16-18], the presence of a temperature gradient also affects the "bulk" Brillouin component of the scattering spectrum, causing an anisotropic asymmetry between the two Brillouin peaks, which has been observed experimentally [19-21]. This asymmetry effect on the Brillouin lines is maximum when $\mathbf{q} \| \boldsymbol{\nabla} T_{0}$ and is zero when $\mathbf{q} \perp \boldsymbol{\nabla} T_{0}$, thus, just the opposite to the nonequilibrium effects on the Rayleigh line. In addition, the presence of a temperature gradient only affects the shape of the Brillouin spectrum; however, it does not affect the total intensity of scattered light, since one of the Brillouin peaks shrinks just the same as the other enhances [18]. In the present paper we are concerned with nonequilibrium fluctuations as can be observed by two experimental techniques (low-angle static light-scattering and shadowgraphy) which probe fluctuations with wave vector $\mathbf{q} \perp \boldsymbol{\nabla} T_{0}$ and which are sensitive to the total intensity of light scattered by the medium. Consequently, nonequilibrium effects on the Brillouin spectrum are not expected to play a role. For this reason and to simplify the calculations, we shall adopt in the present paper hydrodynamic approximations (Boussinesq) which imply that the density fluctuations are only caused by the temperature fluctuations, neglecting the pressure fluctuations. This is equivalent to neglecting the Brillouin components in the scattering spectrum.

An evident shortcoming of Eq. (1) is that such a bulk nonequilibrium structure factor is only valid for negative Rayleigh numbers. For any positive $R$ there is always some finite value of the wave number $q$ for which $S(q)$, as given by Eq. (1), diverges. This shortcoming is a consequence of the fact that in the derivation of Eq. (1) for $S(q)$, boundary effects due to the finite height $L$ of the fluid layer have not been incorporated. Various authors have studied finite-size effects on the nonequilibrium structure factor [22-26]. These investigators have focused their attention exclusively on the situation close to the convective Rayleigh-Bénard instability and studied the divergence of $S(q)$ as the critical value of the Rayleigh number is approached from below.

Finite-size effects on the nonequilibrium structure factor have also been studied recently by Ortiz de Zárate et al., who first considered "stress-free" boundary conditions [27], and subsequently "no-slip" boundary conditions which were evaluated in a first-order Galerkin approximation [28], but without considering the presence of gravity. Hence, the results thus obtained refer to the special case corresponding to $R=0$. It is the purpose of the present paper to consider the boundary effects on the nonequilibrium contribution to the structure factor for both positive and negative Rayleigh numbers.

In a recent paper we have embarked on this program by considering "stress-free" boundary conditions [29]. We were able to derive an explicit expression for the nonequilibrium structure factor valid for both negative and positive values of
$R$ up to the critical Rayleigh number associated with the first convective instability, which for "stress-free" boundary conditions is $R_{c}=27 \pi^{4} / 4$. The main conclusion was that, in the nonequilibrium contribution to the Rayleigh component of the structure factor, finite-size effects cause a crossover from the $q^{-4}$ divergence to a $q^{2}$ dependence for extremely small wave numbers. This crossover from $q^{-4}$ to $q^{2}$ means that a maximum in the Rayleigh-scattering intensity appears. The position of this maximum is close to the critical wave number $q_{c}$ for the appearance of convection. For positive Rayleigh numbers the height of this maximum diverges as the convective instability is approached [29].

The position and height of the maximum in $S(q)$ will depend on the boundary conditions considered. In our previous publication [29] we adopted "stress-free" boundary conditions for the fluctuating velocity because of their mathematical simplicity. But these conditions correspond to a fluid layer bounded by two free surfaces which is rather unrealistic [30]. For the realistic case of a fluid bounded by two rigid solid plates the appropriate boundary conditions are "no-slip" boundary conditions. The goal of the present paper is to analyze the finite-size effects on the nonequilibrium structure factor by considering the more realistic "no-slip" boundary conditions and comparing the results with the finite-size effects obtained with "slip-free" boundary conditions. In doing so, we shall obtain an expression for $S(q)$ for both negative and positive Rayleigh numbers below the critical Rayleigh number for a fluid between rigid walls. By analyzing the behavior of our general expression for $S(q)$ near the convective instability, we shall be able to make contact with expressions and approximations obtained by previous workers [22-26].

We shall proceed as follows. In Sec. II, we present the linearized Boussinesq equations supplemented with random noise terms, which provide the commonly accepted starting point for dealing with thermal nonequilibrium fluctuations in fluids [22,24,31,32], and we also review our previous results obtained for a fluid layer with two free boundaries [29]. Section III contains the main results of the paper, where we consider a fluid layer between two rigid boundaries and adopt a Galerkin-polynomial approximation [28,33]. In Secs. IV and V we study the finite-size effects as they will appear in low-angle light-scattering or shadowgraph experiments, respectively, and we discuss their relevance for the interpretation of available experimental information. In Sec. VI we shall perform a detailed analysis of the nonequilibrium fluctuations close to the convective instability. In Sec. VII we evaluate the so-called power of thermal fluctuations so as to make a detailed numerical comparison with the predictions from the traditional Swift-Hohenberg model. Our conclusions are summarized in Sec. VIII.

\section{LINEARIZED FLUCTUATING BOUSSINESQ EQUATIONS}

We consider a fluid layer between two horizontal plates separated by a distance $L$. The fluid layer is subjected to a temperature gradient in the vertical direction by maintaining the plates at two different temperatures. The size of the sys- 
tem in the two horizontal $X$ - and $Y$ directions is much larger than the size $L$ in the vertical $Z$ direction.

To determine the structure factor of the fluid we consider small fluctuations around the conductive solution. These small fluctuations will be described by the linearized Boussinesq equations supplemented with random noise terms, as first considered by Zaitsev and Shliomis [31] and by Swift and Hohenberg [22] for studying the influence of thermal noise close to the convective instability. Use of the Boussinesq approximation to the full hydrodynamic equations implies that we neglect the sound modes and consider only density fluctuations caused by temperature fluctuations $[24,30]$. We consider the stationary temperature gradient, $\nabla T_{0}$, applied along the $Z$ direction, so that $\nabla T_{0}$ is positive when the fluid layer is heated from above and negative when heated from below. The gravitational force $\mathbf{g}$ is directed in the negative $Z$ direction. In this notation, the Rayleigh number may be defined as

$$
R=\frac{\alpha L^{4} \mathbf{g} \cdot \nabla T_{0}}{\nu D_{T}}=-\frac{\alpha L^{4} g \nabla T_{0}}{\nu D_{T}},
$$

where $\alpha$ is the thermal expansion coefficient and $\nu$ the kinematic viscosity of the fluid.

We shall evaluate the structure factor of the fluid maintained in a convection-free thermal nonequilibrium state, where the average value $\langle\mathbf{v}\rangle$ of the local fluid velocity $\mathbf{v}$ will be zero. Such states correspond to both negative and positive values of the Rayleigh number $R$ as long as $R$ is smaller than the critical value $R_{c}$. For this purpose we write the linearized fluctuating Boussinesq equations in the form $[22,29,32]$

$$
\begin{gathered}
\frac{\partial}{\partial t}\left(\nabla^{2} w\right)=\nu \nabla^{2}\left(\nabla^{2} w\right)+\alpha g\left(\frac{\partial^{2} \theta}{\partial x^{2}}+\frac{\partial^{2} \theta}{\partial y^{2}}\right)+F_{1}, \\
\frac{\partial \theta}{\partial t}=D_{T} \nabla^{2} \theta-w \nabla T_{0}+F_{2},
\end{gathered}
$$

where $\theta=T-T_{0}$ represents the local fluctuating temperature and $w$ is the fluctuating $Z$ component of the fluid velocity $\mathbf{v}$. To eliminate the stationary pressure gradient from the equations we find it convenient to consider Eq. (5a) for $\nabla^{2} w$, rather than an equation for the fluctuating fluid velocity $\mathbf{v}$ itself [30]. Finally, $F_{1}$ and $F_{2}$ represent the contributions from rapidly varying short-range fluctuations and are related to Landau's random stress tensor $\delta \mathrm{T}$ and random heat flow $\delta \mathbf{Q}$ in such a way that [26]

$$
\begin{gathered}
F_{1}=\frac{1}{\rho}\{\boldsymbol{\nabla} \times[\boldsymbol{\nabla} \times(\boldsymbol{\nabla} \cdot \delta \mathbf{T})]\}_{z}, \\
F_{2}=-\frac{D_{T}}{\lambda_{T}} \boldsymbol{\nabla}(\delta \mathbf{Q}),
\end{gathered}
$$

where $\rho$ and $\lambda_{T}$ are the density and the thermal conductivity of the fluid, while the subscript $z$ in Eq. (6a) indicates that $F_{1}$ has to be identified with the $Z$ component of the vector between the curly brackets. We finally note that in the
Boussinesq Eq. (5b) the coefficient multiplying $\nabla^{2} \theta$ is usually identified with the thermal diffusivity of the fluid $D_{T}$. For consistency, we have also expressed the prefactor of the second random noise term in Eq. (6b) in terms of the same diffusivity $D_{T}$ [34].

Since in practice the fluid layer is confined between two horizontal plates separated by a (small) distance $L$, the nonequilibrium structure factor will be affected by the presence of boundary conditions in the $Z$ direction. To accommodate the boundary conditions, we apply a Fourier transformation of the fluctuating Boussinesq equations (5) in space and in time, but restrict the spatial Fourier transformation to the $X-Y$ plane [27-29]. We thus obtain the following set of linear stochastic differential equations:

$$
\left(\begin{array}{cc}
i \omega \mathcal{D}-\nu \mathcal{D}^{2} & \alpha g q_{\|}^{2} \\
\nabla T_{0} & i \omega-D_{T} \mathcal{D}
\end{array}\right)\left(\begin{array}{c}
w\left(\omega, \mathbf{q}_{\|}, z\right) \\
\theta\left(\omega, \mathbf{q}_{\|, z}\right)
\end{array}\right)=\left(\begin{array}{c}
F_{1}\left(\omega, \mathbf{q}_{\|}, z\right) \\
F_{2}\left(\omega, \mathbf{q}_{\|}, z\right)
\end{array}\right),
$$

where $\mathbf{q}_{\|}$is the component of the wave vector $\mathbf{q}$ in the $X-Y$ plane and $\mathcal{D}$ the differential operator

$$
\mathcal{D}=\left[\frac{d^{2}}{d z^{2}}-q_{\|}^{2}\right] .
$$

The random noise terms $F_{1}\left(\omega, \mathbf{q}_{\|}, z\right)$ and $F_{2}\left(\omega, \mathbf{q}_{\|}, z\right)$ in Eq. (7) are related to the partial Fourier transforms $\delta \mathrm{T}\left(\omega, \mathbf{q}_{\|}, z\right)$ of the random stress tensor and $\delta \mathbf{Q}\left(\omega, \mathbf{q}_{\|}, z\right)$ of the random heat flux. The actual expressions are a bit complicated and can be found elsewhere [28].

In this paper we are interested in the structure factor of the nonequilibrium fluid, $S\left(\omega, q_{\|}, z, z^{\prime}\right)$, which is related to the autocorrelation function of the temperature fluctuations by $[27,28]$

$$
\begin{aligned}
\left\langle\theta^{*}\left(\omega, \mathbf{q}_{\|}, z\right) \theta\left(\omega^{\prime}, \mathbf{q}_{\|}^{\prime}, z^{\prime}\right)\right\rangle= & \frac{(2 \pi)^{3}}{\alpha^{2} \rho^{2}} S\left(\omega, q_{\|}, z, z^{\prime}\right) \delta\left(\omega-\omega^{\prime}\right) \\
& \times \delta\left(\mathbf{q}_{\|}-\mathbf{q}_{\|}^{\prime}\right)
\end{aligned}
$$

Integration over the frequency gives the static structure factor $S\left(q_{\|}, z, z^{\prime}\right)=(2 \pi)^{-1} \int d \omega S\left(\omega, q_{\|}, z, z^{\prime}\right)$, which is the main interest of this paper. The result obtained for $S\left(q_{\|}, z, z^{\prime}\right)$ will depend on the boundary conditions at $z=0$ and $z=L$. In a previous publication [29] we calculated, from Eq. (7), $S\left(q_{\|}, z, z^{\prime}\right)$ using stress-free boundary conditions for the vertical velocity and perfectly conducting walls for the temperature. For that purpose, we represented $w\left(\omega, \mathbf{q}_{\|}, z\right)$ and $\theta\left(\omega, \mathbf{q}_{\|}, z\right)$ as a series expansion in a complete set of eigenfunctions of the differential operator in Eq. (7), satisfying the corresponding boundary conditions. Because of the simplicity of the boundary conditions considered in Ref. [29], it was possible to obtain an exact expression for $S\left(q_{\|}, z, z^{\prime}\right)$, with no other simplifications than those contained in the Boussinesq approximation (5). The final results may be expressed as [29] 


$$
\begin{aligned}
S\left(q_{\|}, z, z^{\prime}\right)= & S_{E}\left[\delta\left(z-z^{\prime}\right)+\widetilde{S}_{\mathrm{NE}}^{0} \sum_{N=1}^{\infty} \Lambda_{N}^{F}\left(\tilde{q}_{\|}\right)\right. \\
& \left.\times \sin \left(\frac{N \pi z}{L}\right) \sin \left(\frac{N \pi z^{\prime}}{L}\right)\right],
\end{aligned}
$$

where $\tilde{q}_{\|}=q_{\|} L$. In Eq. (10) $S_{E}$ is the intensity of the fluctuations in thermodynamic equilibrium, given by Eq. (2), and $\widetilde{S}_{\mathrm{NE}}^{0}$ represents the strength of the nonequilibrium enhancement of the structure factor as defined by Eq. (3). To facilitate a comparison with the calculation for the case of rigid boundaries, we have adopted here a definition of $\widetilde{S}_{\mathrm{NE}}^{0}$ in Eq. (3) that is slightly different from the one in our previous publication [29]. Furthermore, we have introduced in Eq. (10) the quantities $\Lambda_{N}^{F}\left(\tilde{q}_{\|}\right)$, which represent the normalized nonequilibrium enhancements per mode and are given by

$$
\Lambda_{N}^{F}\left(\tilde{q}_{\|}\right)=\frac{2}{L} \frac{1}{\sigma+1} \frac{\tilde{q}_{\|}^{2}}{\left(\tilde{q}_{\|}^{2}+N^{2} \pi^{2}\right)^{3}-R \tilde{q}_{\|}^{2}} .
$$

The first term in Eq. (10) is the static structure factor of a fluid in thermodynamic equilibrium; it is short ranged, proportional to a delta function, and it is not affected by any finite-size effects [9]. The second term in Eq. (10) represents the nonequilibrium enhancement of the structure factor. This nonequilibrium enhancement is proportional to $\left(\nabla T_{0}\right)^{2}$ through the expression (3) for $\widetilde{S}_{\mathrm{NE}}^{0}$; it depends on the gravitational acceleration constant $g$ through the appearance of the Rayleigh number in Eqs. (10) and (3), and it depends on the finite height $L$ of the fluid layer explicitly in Eq. (10) and also through $\tilde{q}_{\|}=q_{\|} L$. It is interesting to note that Eq. (10) is valid for both negative and positive Rayleigh numbers, provided that $R<R_{c}=27 \pi^{4} / 4$. For $R \geqslant R_{c}$ there always exist values of $q_{\|}$for which the right-hand side of Eq. (10) diverges. Of course, the value $R_{c}=27 \pi^{4} / 4$ equals the wellknown value obtained form a linear stability analysis of the Boussinesq equations with no-slip boundary conditions [30].

\section{SOLUTION FOR TWO RIGID BOUNDARIES}

While "stress-free" boundary conditions, considered in a previous publication [29], are convenient for obtaining a simple and exact solution of the linearized Boussinesq equations, a fluid bounded by two free surfaces is an unrealistic representation of the actual experimental situation [30,33]. For a fluid layer between two rigid walls we can continue to assume perfectly conducting walls, but we need to adopt "no-slip" boundary conditions for the local velocity. Hence, the boundary conditions to be considered in this paper are

$$
\begin{gathered}
\theta\left(\omega, \mathbf{q}_{\|}, z\right)=0 \quad \text { at } \quad z=0, L, \\
w\left(\omega, \mathbf{q}_{\|}, z\right)=0 \quad \text { at } \quad z=0, L, \\
\frac{d}{d z} w\left(\omega, \mathbf{q}_{\|}, z\right)=0 \quad \text { at } \quad z=0, L .
\end{gathered}
$$

For the boundary conditions (12), the method employed in Ref. [29] to calculate the static structure factor exactly is not adequate. The eigenvalues and eigenfunctions of the differential operator on the left-hand side of Eq. (7) satisfying the new boundary conditions (12) cannot be calculated explicitly. As discussed elsewhere [24,30,33], to calculate the eigenvalues in this case an algebraic equation is obtained which cannot be solved explicitly. The spectrum of the differential operator continues to be discrete, but the set of eigenvalues and eigenfunctions can only be calculated numerically. Hence, for the case of "no-slip" boundary conditions an explicit evaluation of the structure factor can only be performed approximately.

It turns out that a suitable approximation scheme is obtained by using the Galerkin method [28]. Thus we represent the solutions for the velocity fluctuations and temperature fluctuations in terms of first-order Galerkin polynomials,

$$
\begin{gathered}
w\left(\omega, \mathbf{q}_{\|}, z\right)=w_{0}\left(\omega, \mathbf{q}_{\|}\right)\left(\frac{z}{L}-\frac{z^{2}}{L^{2}}\right)^{2}, \\
\theta\left(\omega, \mathbf{q}_{\|}, z\right)=\theta_{0}\left(\omega, \mathbf{q}_{\|}\right)\left(\frac{z}{L}-\frac{z^{2}}{L^{2}}\right) .
\end{gathered}
$$

Note that the polynomials in Eq. (13) satisfy the required boundary conditions (12). The results obtained with this approximation scheme will depend on the adoption of Galerkin test functions. Another possible choice would be the Chandrasekhar function [30]. We have chosen the polynomials (13) because they lead to simpler analytical results and they do not contain constants to be determined numerically. Moreover, in studies of linear stability, the choice (13) is considered to be optimal owing to the variational structure of the underlying problem [33]. Anyway, as we shall see, the choice (13) produces reasonable results when the asymptotic behavior of the structure factor for large $q$ is compared with the behavior expected form the exact "bulk" result [see Eq. (30) below].

Following a standard procedure, we evaluate the amplitudes $\theta_{0}\left(\omega, \mathbf{q}_{\|}\right)$and $w_{0}\left(\omega, \mathbf{q}_{\|}\right)$by imposing the condition that the ansatz (13) represents an exact solution of Eq. (7) in the subspace generated by the corresponding Galerkin polynomial [33]. Substituting Eq. (13) into Eq. (7) and projecting the first equation onto the first Galerkin polynomial and the second equation onto the second Galerkin polynomial, we obtain the set of two algebraic equations,

$$
\mathcal{H}\left(\omega, q_{\|}\right) \cdot\left(\begin{array}{c}
w_{0}\left(\omega, \mathbf{q}_{\|}\right) \\
\theta_{0}\left(\omega, \mathbf{q}_{\|}\right)
\end{array}\right)=\left(\begin{array}{c}
G_{1}\left(\omega, \mathbf{q}_{\|}\right) \\
G_{2}\left(\omega, \mathbf{q}_{\|}\right)
\end{array}\right)
$$

where the matrix $\mathcal{H}\left(\omega, q_{\|}\right)$is given by 


$$
\mathcal{H}\left(\omega, q_{\|}\right)=\left(\begin{array}{cc}
\frac{-i \omega\left(12+q_{\|}^{2} L^{2}\right)}{630 L}-\nu\left[\frac{\left(12+q_{\|}^{2} L^{2}\right)^{2}}{630 L^{3}}+\frac{4}{7 L^{3}}\right] & \frac{\alpha g q_{\|}^{2} L}{140} \\
\frac{\nabla T_{0} L}{140} & \frac{i \omega L}{30}+\frac{D_{T}\left(10+q_{\|}^{2} L^{2}\right)}{30 L}
\end{array}\right)
$$

and where we have introduced the quantities

$$
\begin{aligned}
G_{1}\left(\omega, \mathbf{q}_{\|}\right) & =\int_{0}^{L}\left(\frac{z}{L}-\frac{z^{2}}{L^{2}}\right)^{2} F_{1}\left(\omega, \mathbf{q}_{\|}, z\right) d z, \\
G_{2}\left(\omega, \mathbf{q}_{\|}\right) & =\int_{0}^{L}\left(\frac{z}{L}-\frac{z^{2}}{L^{2}}\right) F_{2}\left(\omega, \mathbf{q}_{\|}, z\right) d z,
\end{aligned}
$$

which are the projections onto the corresponding Galerkin polynomial of the Langevin random noise terms. Upon inverting the matrix $\mathcal{H}\left(\omega, q_{\|}\right)$, the solution of Eq. (14) for the amplitudes $w_{0}(\omega, \mathbf{q} \|)$ and $\theta_{0}(\omega, \mathbf{q} \|)$ can be obtained. Explicit expressions are long and not very informative, so they are not presented here. However, explicit results obtained upon integration of the expression for $\theta_{0}\left(\omega, \mathbf{q}_{\|}\right)$, will be presented in the sequel.

We focus our attention on the calculation of the structure factor, which is related by Eq. (9) to the autocorrelation function of the temperature fluctuations. For the calculation of this quantity, we need the autocorrelation function between the projections of the Langevin random noise terms onto the Galerkin polynomials, defined by Eq. (16). The crosscorrelation $\left\langle G_{1}^{*}\left(\omega, \mathbf{q}_{\|}\right) \cdot G_{2}\left(\omega^{\prime}, \mathbf{q}_{\|}^{\prime}\right)\right\rangle$ is zero, because the random current tensor and the random heat flux are uncorrelated [4]. We now proceed to calculate the autocorrelation functions $\left\langle G_{1}^{*}\left(\omega, \mathbf{q}_{\|}\right) \cdot G_{1}\left(\omega^{\prime}, \mathbf{q}_{\|}^{\prime}\right)\right\rangle$ and $\left\langle G_{2}^{*}\left(\omega, \mathbf{q}_{\|}\right)\right.$ $\left.\cdot G_{2}\left(\omega^{\prime}, \mathbf{q}_{\|}^{\prime}\right)\right\rangle$. We first consider $\left\langle G_{2}^{*}\left(\omega, \mathbf{q}_{\|}\right) \cdot G_{2}\left(\omega^{\prime}, \mathbf{q}_{\|}^{\prime}\right)\right\rangle$ for the random noise associated with the temperature fluctuations. Using the definition of $G_{2}(\omega, \mathbf{q} \|)$, given by Eq. (16), the definition of $F_{2}\left(\omega, \mathbf{q}_{\|}, z\right)$ as a function of the random heat flow, given by Eq. (6b), and the equilibrium correlations between the different components of $\delta \mathbf{Q}\left(\omega, \mathbf{q}_{\|}, z\right)$, as given by Schmitz and Cohen [4], we obtain

$$
\begin{aligned}
\left\langle G_{2}^{*}\left(\omega, \mathbf{q}_{\|}\right) \cdot G_{2}\left(\omega^{\prime}, \mathbf{q}_{\|}^{\prime}\right)\right\rangle= & \frac{2 k_{B} T^{2} \lambda_{T}}{\rho^{2} c_{P}^{2}} \frac{q_{\|}^{2} L^{2}+10}{30 L}(2 \pi)^{3} \\
& \times \delta\left(\omega-\omega^{\prime}\right) \delta\left(\mathbf{q}_{\|}-\mathbf{q}_{\|}^{\prime}\right) .
\end{aligned}
$$

The autocorrelation function $\left\langle G_{1}^{*}\left(\omega, \mathbf{q}_{\|}\right) \cdot G_{1}\left(\omega^{\prime}, \mathbf{q}_{\|}^{\prime}\right)\right\rangle$ has already been evaluated by a similar procedure in a previous publication [28] and is given by

$$
\begin{aligned}
\left\langle G_{1}^{*}\left(\omega, \mathbf{q}_{\|}\right) \cdot G_{1}\left(\omega^{\prime}, \mathbf{q}_{\|}^{\prime}\right)\right\rangle= & 2 k_{B} T \frac{\nu}{\rho} q_{\|}^{2} \frac{q_{\|}^{4} L^{4}+24 q_{\|}^{2} L^{2}+504}{630 L^{3}} \\
& \times(2 \pi)^{3} \delta\left(\omega-\omega^{\prime}\right) \delta\left(\mathbf{q}_{\|}-\mathbf{q}_{\|}^{\prime}\right) .
\end{aligned}
$$

Now we have all the required information to obtain the dynamic structure factor $S\left(\omega, q_{\|}, z, z^{\prime}\right)$ of the fluid. Integration over the frequency $\omega$ gives the static structure factor. After some long, but otherwise straightforward calculations, we find that the static structure factor for the case of "noslip" boundary conditions, in the first-order Galerkin approximation, can be written as

$$
S\left(q_{\|}, z, z^{\prime}\right)=S_{E}\left[\frac{30}{L}+\widetilde{S}_{\mathrm{NE}}^{0} \Lambda_{0}^{\mathrm{R}}\left(\tilde{q}_{\|}\right)\right]\left(\frac{z}{L}-\frac{z^{2}}{L^{2}}\right)\left(\frac{z^{\prime}}{L}-\frac{z^{\prime 2}}{L^{2}}\right),
$$

where, similarly to Eq. (10), we have introduced the quantity $\Lambda_{0}^{\mathrm{R}}\left(\tilde{q}_{\|}\right)$which represents the normalized nonequilibrium enhancement for two rigid boundaries in the first-order Galerkin approximation, and is given by

$$
\Lambda_{0}^{R}\left(\tilde{q}_{\|}\right)=\frac{30}{L} \frac{1}{\sigma+\frac{\left(\tilde{q}_{\|}^{2}+10\right)\left(\tilde{q}_{\|}^{2}+12\right)}{\left(\tilde{q}_{\|}^{2}+12\right)^{2}+360}} \frac{27 \tilde{q}_{\|}^{2}}{28\left(\tilde{q}_{\|}^{2}+10\right)\left[\left(\tilde{q}_{\|}^{2}+12\right)^{2}+360\right]-27 \tilde{q}_{\|}^{2} R} .
$$

In Eq. (19), $S_{E}$ is again the intensity of the thermal fluctuations in thermodynamic equilibrium, defined by Eq. (2), and $\widetilde{S}_{\mathrm{NE}}^{0}$ is the same nonequilibrium enhancement of the structure factor defined by Eq. (3). Equations (19) and (20) for the nonequilibrium structure factor represent our principal re- sults for the combined effects of gravity and finite size on the nonequilibrium structure factor of a fluid between two rigid boundaries. The remainder of this paper will be concerned with an analysis of some of the physical consequences implied by these results. 
It is interesting to compare the approximate solution for the case of two rigid boundaries, given by Eqs. (19) and (20), with the exact solution for the case of two free boundaries, given by Eqs. (10) and (11). First of all, we have obtained here only the first term in the Galerkin expansion for the structure factor for the case of two rigid boundaries. Thus, Eq. (19) is equivalent to the first term in the series expansion (10) for the structure factor of a fluid layer with two free boundaries. Truncating Eq. (10) at $N=1$ is called the mostunstable-mode approximation [22,29,31], and, as we shall see, it represents a good approximation to the exact solution, in particular for small $q$.

As a second comment we note that, when $\nabla T_{0}=0$, the exact solution for two free boundaries, Eq. (10), produces the exact result for the structure factor in equilibrium, which is short ranged and proportional to a delta function $\delta\left(z-z^{\prime}\right)$. However, our approximate solution for two rigid boundaries contains a constant multiplying the Galerkin polynomials. Actually, what we obtain from Eq. (19) for the equilibrium structure factor is the first term of the series expansion of the delta function in terms of the Galerkin polynomials. Therefore, this shortcoming is a consequence of having performed the calculation in first order only.

The normalized nonequilibrium enhancement for the case of two rigid boundaries in first order, $\Lambda_{0}^{R}\left(\tilde{q}_{\|}\right)$, has a structure very similar to the general term $\Lambda_{N}^{F}\left(\tilde{q}_{\|}\right)$, given by Eq. (11), for the case of two free boundaries. With regards to the dependence on the Prandtl number, the 1 in the denominator of the term $1 /(\sigma+1)$, appearing in Eq. (11) for two free boundaries, is replaced with a rational function of $\tilde{q}_{\|}$, which rapidly approaches unity as $\tilde{q}_{\|}$increases. With regards to the dependence on the Rayleigh number, this is also very similar: the factor $\left(\tilde{q}_{\|}^{2}+N^{2} \pi^{2}\right)^{3}$ in Eq. (11) for two free boundaries is simply replaced with a polynomial of sixth order in $\tilde{q}_{\|}$.

The approximate solution for two rigid boundaries breaks down when

$$
R=\frac{28}{27} \frac{\left(\tilde{q}_{\|}^{2}+10\right)\left[\left(\tilde{q}_{\|}^{2}+12\right)^{2}+360\right]}{\tilde{q}_{\|}^{2}} .
$$

In Eq. (21) we recognize the threshold condition for the convective instability, as calculated by the Galerkin method in first order [33], and we recover the well-known results of linear stability theory from studying the divergences of the structure factor, which are associated with the appearance of convection. For Eq. (21) to hold, the Rayleigh number $R$ has to be larger than a critical Rayleigh number $R_{c} \simeq 1750$. For this critical Rayleigh number, Eq. (21) yields [33] $\tilde{q}_{c}$ $\simeq 3.1165$. These critical values in the first Galerkin approximation are to be compared with the exact threshold values for the case of two rigid boundaries, which are [33] $R_{c}$ $\simeq 1708$ and $\tilde{q}_{c} \simeq 3.1163$. We note again that introduction of boundary conditions in the calculation of the structure factor, results in an extension of the validity of Eq. (1) for the structure factor at negative Rayleigh numbers to a finite range of positive Rayleigh numbers. For the case of two rigid bound- aries, the interval of positive $R$ for which it is possible to calculate the structure factor with a linear theory is larger than for the case of two free boundaries. This is a consequence of the fact that "no-slip" boundary conditions are more stabilizing than "stress-free" boundary conditions.

\section{CONSEQUENCES FOR LIGHT-SCATTERING EXPERIMENTS}

The nonequilibrium fluctuations can be detected in smallangle light-scattering experiments [10-15]. The scattering medium in such experiments is a thin horizontal fluid layer bounded by two parallel plates whose temperatures can be controlled independently so as to establish a temperature gradient across the fluid layer. The temperature gradient can be parallel or antiparallel to the direction of gravity. The horizontal plates are furnished with windows allowing laser light to propagate through the fluid in the direction parallel to the gravity and to the temperature gradient. Light scattered over an angle $\phi$ arises from fluctuations with a wave number such that [9]: $q=2 q_{0} \sin (\phi / 2)$, where $q_{0}$ is the wave number of the incident light inside the scattering medium. To observe any nonequilibrium fluctuations one needs to observe the scattered light at small wave numbers and, hence, at small scattering angles.

From electromagnetic theory [9] it follows that the scattering intensity $S(\mathbf{q})$ is obtained from an integration of the structure factor over the scattering volume. When the wave vector of the incident light is parallel to the temperature gradient and for very small scattering angles (scattering vector $\mathbf{q} \perp \boldsymbol{\nabla} T_{0}$ ), the total intensity of scattered light is proportional to $[24,27,29]$

$$
S\left(q_{\|}, q_{\perp}\right)=\frac{1}{L} \int_{0}^{L} \int_{0}^{L} e^{-i q_{\perp}\left(z-z^{\prime}\right)} S\left(q_{\|}, z, z^{\prime}\right) d z d z^{\prime},
$$

where it is assumed [24] that the scattering volume extends over the full height of the fluid layer and that the thickness of the scattering volume $L_{s}$ is larger than $q_{\|}^{-1}$. In an actual light-scattering experiment the magnitude of the components $q_{\|}$and $q_{\perp}$ of the scattering vector are not independent variables, they are both related to the scattering angle [27,29] and for the interpretation of small-angle experiments one may use in practice the approximation $q_{\|} \simeq q, q_{\perp} \simeq 0$. Hence, the nonequilibrium structure factor $S\left(q_{\|}, q_{\perp}\right)$, defined by Eq. (22), depends only on the magnitude $q$ of the scattering wave vector q. In the remainder of this paper we restrict ourselves to this small-angle approximation.

To obtain the exact expression for the structure factor in the small-angle approximation, $S\left(q_{\|} \simeq q, q_{\perp} \simeq 0\right)$, for the case of two free boundaries, we substitute Eq. (10) into Eq. (22) and perform the double integration in Eq. (22). Introducing a dimensionless wave number $\tilde{q}=q L$, we thus obtain

$$
S(\tilde{q})=S_{E}\left[1+\widetilde{S}_{\mathrm{NE}}^{0} \widetilde{S}_{\mathrm{NE}}^{F}(\tilde{q})\right],
$$

where we have introduced the normalized nonequilibrium enhancement for free boundaries, $\widetilde{S}_{\mathrm{NE}}^{F}(\tilde{q})$, such that 


$$
\widetilde{S}_{\mathrm{NE}}^{F}(\tilde{q})=\sum_{N=1}^{\infty} \Lambda_{N}^{F}(\tilde{q}) \frac{2 L[1-\cos (N \pi)]}{N^{2} \pi^{2}}
$$

with the nonequilibrium enhancement per mode, $\Lambda_{N}^{F}(\tilde{q})$, given by Eq. (11) with $\tilde{q}=\tilde{q}_{\|}$.

To obtain the nonequilibrium structure factor at small scattering angles for the case of two rigid boundaries, we substitute Eq. (10) into Eq. (22) and perform the double integration in Eq. (22), to obtain

$$
S(\tilde{q})=S_{E}\left[\frac{5}{6}+\widetilde{S}_{\mathrm{NE}}^{0} \widetilde{S}_{\mathrm{NE}}^{R}(\tilde{q})\right],
$$

where the normalized nonequilibrium enhancement for rigid boundaries, $\widetilde{S}_{\mathrm{NE}}^{R}(\tilde{q})$ is given by

$$
\widetilde{S}_{\mathrm{NE}}^{R}(\tilde{q})=\frac{L}{36} \Lambda_{0}^{R}(\tilde{q})
$$

with the first-order nonequilibrium enhancement, $\Lambda_{0}^{R}(\tilde{q})$, given by Eq. (20) with $\tilde{q}=\tilde{q}_{\|}$. We note that for the case of two free boundaries the exact $S(\tilde{q})$, given by Eq. (23), is written as the sum of an isotropic equilibrium contribution, $S_{E}$, and a nonequilibrium contribution which is expressed in terms of a series expansion through Eq. (24).

For the case of rigid boundaries, Eqs. (25) and (26) represent the first Galerkin approximation in which the equilibrium contribution is $17 \%(=1 / 6)$ lower than the actual value, while the expression (26) obtained for the nonequilibrium enhancement is the first term of a series expansion. In principle, better results can be obtained, both for the equilibrium and the nonequilibrium contributions for rigid boundaries, by considering higher-order Galerkin approximants.

The exact expression for the normalized nonequilibrium enhancement for two free boundaries, $\widetilde{S}_{\mathrm{NE}}^{R}(\tilde{q})$ was studied in a previous publication, where the sum of the series in Eq. (24) was calculated exactly [29]. We showed that in the limit $L \rightarrow \infty$ the sum of the series in Eq. (24) converges to the exact "bulk" result: $(\sigma+1)^{-1}\left(\tilde{q}^{4}-R\right)^{-1}$ [cf., Eq. (1)]. The dependence of the nonequilibrium enhancement on the dimensionless wave number $\tilde{q}$ was analyzed in detail. For small $\tilde{q}$ we obtained [29]

$$
\widetilde{S}_{\mathrm{NE}}^{F}(\tilde{q}) \stackrel{\tilde{q} \rightarrow 0}{\longrightarrow} \frac{17}{20160} \frac{1}{\sigma+1} \tilde{q}^{2},
$$

and for large $\tilde{q}$

$$
\widetilde{S}_{\mathrm{NE}}^{F}(\tilde{q}) \stackrel{\tilde{q} \rightarrow \infty}{\longrightarrow} \frac{1}{\sigma+1} \frac{1}{\tilde{q}^{4}} .
$$

Equations (27) and (28) demonstrate the crossover from $q^{-4}$ to a $q^{2}$ behavior. Separating the two limiting behaviors there is a maximum in $\widetilde{S}_{\mathrm{NE}}^{F}(\tilde{q})$. For negative $R$ this maximum is relatively flat, while for positive $R$ the maximum is very

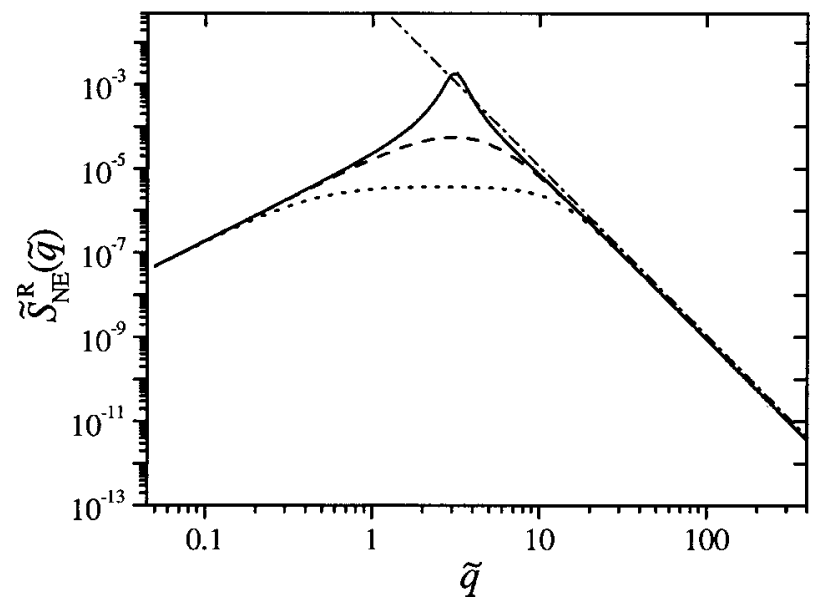

FIG. 1. First-order Galerkin approximation for the nonequilibrium enhancement $\widetilde{S}_{\mathrm{NE}}^{R}(\tilde{q})$, given by Eq. (26) of a fluid with Prandtl number $\sigma=8$, as a function of the dimensionless wave number $\tilde{q}$ $=q L$ for three values of the Rayleigh number: $R=1700$ (solid curve), $R \simeq 0$ (dashed curve), $R=-25000$ (dotted curve). For reference, the correct asymptotic "bulk" behavior, given by Eq. (28), is displayed as a dashed-dotted line.

sharp. The height of the maximum diverges as $R \rightarrow R_{c}$. For details and further comments, we refer to our previous publication [29].

Similarly, from the first-order Galerkin approximation for the nonequilibrium enhancement with rigid boundaries, Eq. (26), we obtain for small $\tilde{q}$,

$$
\widetilde{S}_{\mathrm{NE}}^{R}(\tilde{q}) \stackrel{\tilde{q} \rightarrow 0}{\longrightarrow} \frac{3}{896(21 \sigma+5)} \tilde{q}^{2},
$$

and for large $\tilde{q}$,

$$
\widetilde{S}_{\mathrm{NE}}^{R}(\tilde{q}) \stackrel{\tilde{q} \rightarrow \infty}{\longrightarrow} \frac{45}{56} \frac{1}{\sigma+1} \frac{1}{\tilde{q}^{4}} .
$$

Just as for two free boundaries, the nonequilibrium enhancement exhibits a crossover from a $q^{-4}$ to a $q^{2}$ behavior. Upon comparing Eq. (28) with the asymptotic behavior corresponding to the "bulk" solution, Eq. (1), we note that the solution for free boundaries reproduces for large $q$ the correct limiting behavior, which is independent of the Rayleigh number. The first-order Galerkin approximation for rigid boundaries reproduces this asymptotic behavior for large $q$, except for a factor $45 / 56$.

In Fig. 1 we show on a double-logarithmic scale the nonequilibrium enhancement $\widetilde{S}_{\mathrm{NE}}^{R}(\tilde{q})$ as a function of $\tilde{q}$, calculated from Eq. (26) for three different values of the Rayleigh number. In all examples to be presented in this paper, we use a value of the Prandtl number $\sigma=8$, which approximately corresponds to that of pure toluene at $20^{\circ} \mathrm{C}$ [12]. The solid curve corresponds to a positive Rayleigh number $R=1700$, to be compared with the critical Rayleigh number $R_{c}$ $\simeq 1750$ in the first-order Galerkin approximation. The dashed curve corresponds to a value of the Rayleigh number close to 
zero: $R \simeq 0$. The dotted curve corresponds to a large negative Rayleigh number $R=-25000$. The dashed-dotted straight line in Fig. 1 shows the exact asymptotic behavior for large $\tilde{q},(\sigma+1)^{-1} \tilde{q}^{-4}$ as given by Eq. (1) for the structure factor of the bulk fluid. From the results displayed in Fig. 1 we observe that at larger values of $\tilde{q}, \widetilde{S}_{\mathrm{NE}}^{R}(\tilde{q})$ is proportional to $\tilde{q}^{-4}$, independent of the Rayleigh number, in agreement with Eq. (29). On the scale used in Fig. 1, $\widetilde{S}_{\mathrm{NE}}^{R}(\tilde{q})$ is asymptotically almost indistinguishable from the exact asymptotic behavior, confirming that the Galerkin calculation is an excellent approximation method. Upon decrease of $\tilde{q}, \widetilde{S}_{\mathrm{NE}}^{R}(\tilde{q})$ goes through a maximum and for very small values of $\tilde{q}$, $\widetilde{S}_{\mathrm{NE}}(\tilde{q})$ decreases as $\tilde{q}^{2}$, also independently of $R$, in agreement with Eq. (29). For positive $R, \widetilde{S}_{\mathrm{NE}}^{R}(\tilde{q})$ develops a prominent peak close to $\tilde{q}_{c} \simeq 3.12$, which diverges as $R$ $\rightarrow R_{c}$, as will be further discussed in Sec. VI. For negative $R$, the maximum is relatively flat, in accordance with the saturation effect of gravity on the nonequilibrium enhancement, already contained in expression (1) for the structure factor of the fluid without any finite-size effects. Although there are some numerical differences, the physical behavior of $\widetilde{S}_{\mathrm{NE}}^{R}(\tilde{q})$ as a function of the wave number and the Rayleigh number is similar to that earlier deduced for the case of free boundaries [29]. Regardless of the detailed nature of the boundary conditions, a major effect of the additive noise terms in the fluctuating Boussinesq equations is the appearance of (fluctuating) patterns in the fluid, even below the convective instability as discussed by some other investigators $[35,36]$.

Sengers and co-workers have measured the nonequilibrium fluctuations in liquid toluene $[10,11]$ and in liquid $n$-hexane [12]. These experiments correspond to Rayleigh numbers from -25000 to -300000 at dimensionless wave numbers ranging from $\tilde{q}=640$ down to $\tilde{q}=345$. The experiments have provided an accurate confirmation of the $q^{-4}$ dependence of the intensity of nonequilibrium fluctuations in this range of wave numbers.

Giglio and co-workers have measured the intensity of nonequilibrium fluctuations for negative Rayleigh numbers down to wave numbers $\tilde{q}$ of order unity with ultra-low-angle light-scattering experiments $[14,15]$. They actually measured the intensity of nonequilibrium concentration fluctuations in a liquid mixture. However, due to the similar structure of the underlying hydrodynamic equations, the $q$ dependence of the contribution of nonequilibrium concentration fluctuations to the structure factor in a liquid mixture is expected to be similar to the $q$ dependence of the contribution of nonequilibrium temperature fluctuations to the structure factor of a one-component fluid [37,38]. Giglio and co-workers have not only confirmed the $q^{-4}$ dependence of the nonequilibrium structure factor, but they have also observed the crossover to a region of $\tilde{q}$ close to unity where the nonequilibrium structure factor is independent of $\tilde{q}$, in agreement with the flat range indicated in Fig. 1 for $\widetilde{S}_{\mathrm{NE}}^{R}(\tilde{q})$ at large negative Rayleigh numbers.

Before closing this section, we note that the scattered in- tensity will be actually proportional to $S(q)$, as given by Eq. (22), only for $q \gg L_{s}^{-1}$, where $L_{s}$ was the thickness of the scattered volume, or, in the geometry we are considering, the thickness of the laser beam. For extremely small $q$, effects related to the small thickness of the beam, not discussed in this paper, are expected to show up. These effects could hamper the actual observation by small-angle light-scattering experiments of the finite-size effects discussed in this section. Fortunately, there is another experimental technique, namely, quantitative shadowgraphy, which is more suitable for the observation of effects on the nonequilibrium structure factor due to the finite size $L$ of the layer, and which we discuss in the subsequent section.

\section{CONSEQUENCES FOR SHADOWGRAPH EXPERIMENTS}

An alternative promising experimental technique for measuring the intensity of nonequilibrium fluctuations is quantitative shadowgraph analysis [39-43]. Instead of a laser beam, an extended uniform monochromatic light source is employed to illuminate the fluid layer. Then many shadowgraph images of a plane perpendicular to the temperature gradient are obtained with a charge-coupled device detector, which measures the spatial distribution of intensity $I\left(\mathbf{x}_{\|}\right)$, where $\mathbf{x}_{\|}$is a two-dimensional position vector in the imaging plane. For each image, a shadowgraph signal $\mathcal{I}\left(\mathbf{x}_{\|}\right)$is defined by

$$
\mathcal{I}\left(\mathbf{x}_{\|}\right)=\frac{I\left(\mathbf{x}_{\|}\right)-I_{0}}{I_{0}}
$$

where $I_{0}$ is the uniform intensity of the incident light, when there are no fluctuations in the index of refraction of the sample. In practice, $I_{0}$ is obtained by averaging over many shadowgraph pictures. Very recently, Trainoff and Cannell [44] have presented a detailed theoretical analysis of the quantitative shadowgraph method based on physical optics. They studied not only the shadowgraph images produced by fluctuations below the convective threshold, but also the shadowgraph images produced by deterministic patterns above the threshold. With a paraxial approximation for the propagation of light in the shadowgraph medium and a Fresnel approximation for the propagation of light in the air behind the cell, the spatial power spectrum of the shadowgraph signal averaged over fluctuations, $\left\langle\left|\mathcal{I}\left(\mathbf{q}_{\|}\right)\right|^{2}\right\rangle$, can be related to the structure factor as defined by Eq. (22), such that $[43,44]$,

$$
\left\langle\left|\mathcal{I}\left(\mathbf{q}_{\|}\right)\right|^{2}\right\rangle=\frac{4 V}{\alpha^{2} \rho^{2}}\left(\frac{\partial n}{\partial T}\right)_{P}^{2} \sin ^{2}\left(\frac{q_{\|}^{2} z}{2 k_{0}}\right) S\left(q_{\|}, 0\right),
$$

where $V$ is the sample volume illuminated by the light. In Eq. (32), the sine term plays the role of an optical transfer function [43]. Trainoff and Cannell [44] have also evaluated small modifications to Eq. (32) due to experimental effects such as inhomogeneities in the illumination, angular spread in the incident beam, or finite spectral bandwidth of the light source. 


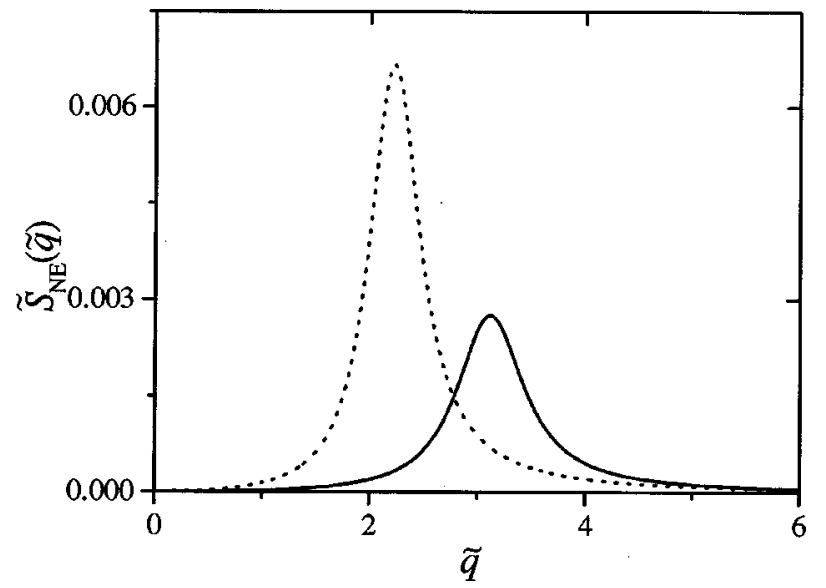

FIG. 2. Nonequilibrium enhancement of the structure factor as a function of $\tilde{q}$ for positive Rayleigh number corresponding to $\epsilon$ $=\left(R-R_{c}\right) / R_{c}=-0.02$. Solid curve: $\widetilde{S}_{\mathrm{NE}}^{R}(\tilde{q})$, given by Eq. $(26)$, for the case of two rigid boundaries. Dashed curve: exact $\widetilde{S}_{\mathrm{NE}}^{F}(\tilde{q})$, given by Eq. (24), for the case of two free boundaries. The plots are for a fluid with Prandtl number $\sigma=8$.

We observe that, by applying a two-dimensional Fourier transform to the shadowgraph signal $[40,41,43]$, one can deduce the structure factor of the fluid as a function of the wave number $q$ at $q_{\perp}=0$. Hence, there exists an equivalence between small-angle light scattering and shadowgraphy, in the sense that both methods yield $S\left(q_{\|}=q, q_{\perp}=0\right)$. For light scattering, $q$ is the scattering wave number, whereas for shadowgraphy $q$ is the modulus of the two-dimensional Fourier vector in the imaging plane. Thus, the characteristic features of the nonequilibrium enhancement of the structure factor, shown in Fig. 1, also apply to shadowgraph experiments. Specifically, Vailati and Giglio [42] and Brogioli et al. $[43,45]$ have used the shadowgraph technique to measure the structure factor of the nonequilibrium fluctuations in some aqueous solutions, resulting from concentration gradients associated with free diffusion. Indeed, the experimental structure factors exhibit the same characteristic features as the structure factor of the nonequilibrium fluctuations in a binary liquid mixture subjected to a concentration gradient resulting from an imposed temperature gradient as measured by light scattering $[14,15]$.

For positive Rayleigh numbers, $\widetilde{S}_{\mathrm{NE}}(\tilde{q})$ strongly depends on the parameter $\epsilon=\left(R-R_{c}\right) / R_{c}$ which measures the distance from the Rayleigh-Bénard instability. In Fig. 2 we show the normalized nonequilibrium enhancement as a function of $\tilde{q}$ close to $\tilde{q}_{c}$ for two rigid boundaries, as calculated from Eq. (26) for $\epsilon=-0.02$, relative to the critical Rayleigh number $R_{c}^{R} \simeq 1750$ in the first-order Galerkin approximation. For comparison, we also show in Fig. 2 the normalized nonequilibrium enhancement as a function of $\tilde{q}$ for two free boundaries, as calculated from Eq. (24) for the same $\epsilon$ $=-0.02$, relative to the critical Rayleigh number $R_{c}^{F}$ $=27 \pi^{4} / 4$ for free boundaries. We observe that the main difference between the solutions for free and rigid boundaries is that for the case of free boundaries the position of the maximum is incorrectly displaced to lower values of $\tilde{q}$. In addi-

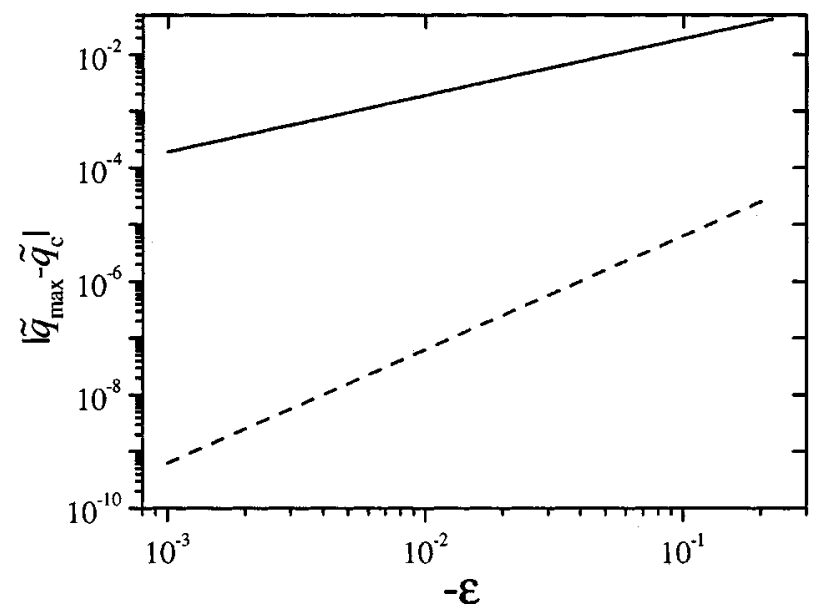

FIG. 3. Double-logarithmic plot of the absolute value of the difference between the critical wave number $\tilde{q}_{c}$ and the position of the maximum in the nonequilibrium structure factor $\tilde{q}_{\max }$, as a function of $-\epsilon$. The solid line is for the case of two rigid boundaries. The dashed line is for the case of two free boundaries. The plots are for a fluid with Prandtl number $\sigma=8$.

tion, we note that the height of the maximum is larger for the case of two free boundaries. As mentioned earlier, "no-slip" boundary conditions are more realistic for a fluid layer between two rigid plates.

Wu et al. [39] have used the shadowgraph technique to measure the nonequilibrium structure factor in a layer of fluid carbon dioxide, at a pressure of about $3 \mathrm{MPa}$, near the convective instability. Figure 3 in the paper of Wu et al. [39] shows an experimental structure factor $S(q)$ with a shape very similar to our solution displayed in Fig. 2. We shall make a quantitative comparison with the measurements of Wu et al. in Sec. VII, when discussing the power of thermal fluctuations. We conclude that our solution of the linearized fluctuating Boussinesq equations for the nonequilibrium structure factor is consistent with the characteristic features of the nonequilibrium structure factor observed in experiments for both negative and positive Rayleigh numbers.

\section{NONEQUILIBRIUM FLUCTUATIONS CLOSE TO THE CONVECTIVE INSTABILITY}

The nature of thermal noise near the convective instability has been the subject of studies by many investigators [22$26,31]$. Hence, it is of interest to make a comparison of those results with our solution for the intensity of temperature fluctuations for thermal nonequilibrium states. Zaitsev and Shliomis [31] were the first to compute thermal fluctuations in a fluid layer subjected to a stationary temperature gradient near the convective instability. Using linear perturbation theory they found that the structure factor diverges as $\left(R_{c}\right.$ $-R)^{-1}$. The same divergence follows from our solutions, both for the case of free boundaries and for the case of rigid boundaries. To reproduce this divergence we first calculate the wave numbers, $\tilde{q}_{\max }^{F}$ and $\tilde{q}_{\max }^{R}$, of the fluctuations that are maximally enhanced for the case of free boundaries and for the case of rigid boundaries, respectively. The quantity $\tilde{q}_{\max }^{F}$ was already evaluated in a previous publication [29], where we showed that it has an expansion of the form 


$$
\begin{aligned}
\tilde{q}_{\max }^{F} & =\tilde{q}_{c}^{F}\left\{1+\frac{81}{4} \sum_{N=2}^{\infty} \frac{\left(N^{2}-1\right)\left(1+2 N^{2}\right)^{2}(1-\cos N \pi)}{N^{2}\left[\left(1+2 N^{2}\right)^{3}-27\right]^{2}} \epsilon^{2}+O\left(\epsilon^{3}\right)\right\} \\
& \simeq \frac{\pi}{\sqrt{2}}\left\{1+2.847 \times 10^{-4} \epsilon^{2}\right\} .
\end{aligned}
$$

For the case of two rigid boundaries, we find from from Eq. (20) that the position of the maximum, $\tilde{q}_{\max }^{R}$, close to the instability may be expanded as

$$
\begin{aligned}
\tilde{q}_{\max }^{R} & =\tilde{q}_{c}^{R}\left\{1+\frac{14}{27} \frac{\left(\tilde{q}_{c}^{2}+10\right)^{2}}{R_{c}^{R} \tilde{q}_{c}^{2}\left(3 \tilde{q}_{c}^{2}+34\right)} \frac{\tilde{q}_{c}^{4}+384 \tilde{q}_{c}^{2}+4104}{\tilde{q}_{c}^{2} \sigma+5} \epsilon+O\left(\epsilon^{2}\right)\right\} \\
& \simeq 3.1165\left\{1+\frac{0.154}{\sigma+0.515} \epsilon\right\}
\end{aligned}
$$

where, inside the curly brackets in Eq. (34), the critical wave number $\tilde{q}_{c}^{R} \simeq 3.1165$ corresponds to the first-order Galerkin approximation for two rigid boundaries. It is interesting to note that the difference between the wave number $\tilde{q}_{\max }$ corresponding to the maximum and the critical wave number $\tilde{q}_{c}$ has a quadratic dependence on the distance $\epsilon$ to the instability for the case of free boundaries, while this difference depends linearly on $\epsilon$ for the case of two rigid boundaries. In the case of free boundaries, the maximum in the structure factor moves to larger wave numbers as $\epsilon$ goes to lower negative values (away from the instability). In the case of rigid boundaries, the maximum in the structure factor moves to smaller wave numbers as $\epsilon$ goes to lower negative values. In Fig. 3, we have plotted on a double-logarithmic scale the absolute value of the difference $\left|\tilde{q}_{\max }-\tilde{q}_{c}\right|$ as a function of $-\epsilon$ for two rigid boundaries (solid line) and for two free boundaries (dashed line). The curves displayed in Fig. 3 were obtained numerically from Eqs. (24) and (26), confirming the linear dependence on $\epsilon$ for rigid boundaries as opposed to a quadratic dependence on $\epsilon$ for free boundaries. Even more significantly, the effect is orders of magnitude larger for rigid boundaries than that for free boundaries.

It is interesting to note that the wave number $\tilde{q}_{\max }$ corresponding to the maximum intensity of the fluctuations cannot be identified with the wave number $\tilde{q}_{m}$ corresponding to the maximum growth rate of perturbations around the steady conductive state evaluated by other investigators [46-48], although both become equal to $\tilde{q}_{c}$ at $R=R_{c}$. This issue is discussed in more detail in the Appendix.

Having determined the position of the maximum, we can study the divergence in the height of the maximum as the convective instability is approached. For the case of two free boundaries, we substitute Eq. (33) into Eq. (24) and conclude that the structure factor, which is proportional to the intensity of the scattered light, diverges when the convective instability is approached, such that

$$
\begin{aligned}
\widetilde{S}_{\mathrm{NE}}^{F}\left(\tilde{q}_{\max }\right)= & \frac{1}{\sigma+1}\left\{54 \pi^{2} \frac{-1}{\epsilon} \frac{16}{\pi^{6}} \sum_{N=2}^{\infty} \frac{1-\cos N \pi}{N^{2}\left[\left(1+2 N^{2}\right)^{3}-27\right]}\right. \\
& +O(\epsilon)\} .
\end{aligned}
$$

For the case of two rigid boundaries, substituting Eq. (34) into Eq. (26) we conclude that the structure factor, in the first-order Galerkin approximation, diverges when the convective instability is approached, such that

$$
\widetilde{S}_{\mathrm{NE}}^{R}\left(\tilde{q}_{\max }\right)=\frac{1}{\sigma+\frac{5}{\tilde{q}_{c}^{2}}}\left\{\frac{5}{6 R_{c}^{R}} \frac{-1}{\epsilon}+O(1)\right\} .
$$

In both cases, we recover the linear divergence of $\widetilde{S}_{\mathrm{NE}}$ as a function of $\left(R-R_{c}\right)^{-1}$ obtained by Zaitsev and Shilomis [31] and confirmed by Swift and Hohenberg [22,26]. Extremely close to the instability nonlinear effects will cause a smearing out of the transition, but this effect will only be noticeable for very small values of $|\epsilon| \leqslant 2.9 \times 10^{-5}$ $[22,26,49-51]$. Hence, observation of the linear divergence of the intensity of the fluctuations is possible in experiments [39]. Deviations from linear fluctuation theory have been observed by Scherer et al. [52] in the case of electroconvection.

For the case of two free boundaries, the approximation scheme used by Zaitsev and Shliomis [31] and by Swift and Hohenberg [22] is equivalent to retaining only the term $N$ $=1$ in the series expansion $(24)$ for $\widetilde{S}_{\mathrm{NE}}^{F}(\tilde{q})$. Note that, for $N=1$, when $R$ is close to $R_{c}$ and $q_{\|}$is close to $q_{c}$, the denominator in the term $\Lambda_{1}^{F}\left(\tilde{q}_{\|}\right)$given by Eq. (11) approaches zero. Therefore, close to the convective instability the term with $N=1$ is much larger than the terms with any other value of $N$. Consequently, when $R \leq R_{c}$ and $q \simeq q_{c}$, truncating the series (24) at $N=1$ yields a very good approximation. We thus deduce from our solution for the case 
TABLE I. Values of the parameters in the SH approximation, Eq. (39), for the nonequilibrium structure factor.

\begin{tabular}{lcccc}
\hline \hline & $R_{c}$ & $\tilde{q}_{c}$ & $\widetilde{\xi}_{0}^{2}$ & $\tilde{H}(\sigma)$ \\
\hline Free $^{\mathrm{a}}$ & $\frac{27 \pi^{4}}{4}=657$ & $\frac{\pi}{\sqrt{2}}=2.221$ & $\frac{2}{\sqrt{3} \pi^{2}}=0.117$ & $\frac{8}{\pi^{2}} \frac{1}{\sigma+1}$ \\
Rigid $^{\mathrm{b}}$ & 1750 & 3.1165 & $\frac{1}{\tilde{q}_{c}} \sqrt{\frac{28\left(3 \tilde{q}_{c}^{2}+34\right)}{27 R_{c}}}=0.062$ & $\frac{5}{6} \frac{\tilde{q}_{c}^{2}}{\tilde{q}_{c}^{2} \sigma+5}$ \\
Rigid $^{\mathrm{c}}$ & 1708 & 3.1163 & 0.062 & \\
\hline \hline
\end{tabular}

${ }^{a}$ The values for two free boundaries are exact.

${ }^{b}$ Values based on a first-order Galerkin approximation.

${ }^{c}$ Exact values obtained from the literature [26].

of free boundaries that the nonequilibrium structure factor, as measured in low-angle light scattering or shadowgraph experiments, can be written as

$$
S^{F}(\tilde{q})=\frac{8 S_{E} \widetilde{S}_{\mathrm{NE}}^{0}}{\pi^{2}(\sigma+1)} \frac{\tilde{q}^{2}}{\left(\tilde{q}^{2}+\pi^{2}\right)^{3}-R \tilde{q}^{2}},
$$

where the equilibrium contribution in Eq. (23) has been neglected. This is usually called the most-unstable-mode approximation in the literature [22,31]. Because of the factor $N^{2} \pi^{2}$ in the denominator of the series expansion (24), the most-unstable-mode approximation actually continues to be an excellent approximation for arbitrary Rayleigh numbers, especially for relatively small $\tilde{q}$. For the case of rigid boundaries, the first-order Galerkin approximation, Eq. (26), already represents a most-unstable-mode approximation. Neglecting the equilibrium contribution, as was done in Eq. (37), we obtain from Eqs. (20) and (26) for rigid boundaries,

$$
\begin{aligned}
S^{R}(\tilde{q})= & \frac{S_{E} \widetilde{S}_{\mathrm{NE}}^{0}}{\sigma+\frac{\left(\tilde{q}^{2}+10\right)\left(\tilde{q}^{2}+12\right)}{\left(\tilde{q}^{2}+12\right)^{2}+360}} \\
& \times \frac{27 \widetilde{q}^{2}}{28\left(\tilde{q}^{2}+10\right)\left[\left(\tilde{q}^{2}+12\right)^{2}+360\right]-27 \tilde{q}^{2} R} .
\end{aligned}
$$

Comparing Eq. (37) for free boundaries with Eq. (38) for rigid boundaries, the similar structure of both expressions is evident, as was already mentioned in Sec. III.

The denominator in the last term of Eq. (37) for free boundaries, as well as the denominator in the last term of Eq. (38) for rigid boundaries, are zero when the Rayleigh number and the dimensionless wave number are equal to the corresponding critical values, $R=R_{c}$ and $\tilde{q}=\tilde{q}_{c}$. Expanding, in both cases, the denominator in powers of $\widetilde{q}^{2}$ around $\tilde{q}_{c}^{2}$, one obtains

$$
S(\tilde{q})=\frac{S_{E} \widetilde{S}_{\mathrm{NE}}^{0}}{R_{c}} \frac{\tilde{H}(\sigma)}{\widetilde{\xi}_{0}^{4}\left(\widetilde{q}^{2}-\widetilde{q}_{c}^{2}\right)^{2}-\epsilon},
$$

where we have introduced the function $\widetilde{H}(\sigma)$ of the Prandtl number and, following Hohenberg and Swift [26], the parameter $\widetilde{\xi}_{0}^{2}$ (see Table I). Equation (39) is the so-called SwiftHohenberg ( $\mathrm{SH}$ ) approximation to the nonequilibrium structure factor $[22,26]$. It is worth noting that Eq. (39) is valid for both free or rigid boundaries, but the numerical values of the constants $R_{c}, q_{c}$, and $\widetilde{\xi}_{0}^{2}$, and the amplitude function $\widetilde{H}(\sigma)$, do depend on the boundary conditions. In the first two rows of Table I we present the values obtained for the constants and for the function $\widetilde{H}(\sigma)$ in the solution of the linearized fluctuating Boussinesq equations for free and rigid boundaries. In the third row of Table I we present, for the case of rigid boundaries, the numerical values of the parameters $R_{c}$, $\tilde{q}_{c}$, and $\widetilde{\xi}_{0}^{2}$, obtained from the literature [26]. In the case of free boundaries, we find exact analytical agreement between the values of $R_{c}, \tilde{q}_{c}$, and $\tilde{\xi}_{0}^{2}$ displayed in Table I and the corresponding literature values [26]. For the case of rigid boundaries, comparing the second and the third rows of Table I, we see a fairly good agreement between the numerical literature values and the analytical values obtained here based on a first-order Galerkin approximation.

\section{POWER OF THERMAL FLUCTUATIONS}

To further compare our results with the SH model, we consider the behavior of the vertical average of the power of the thermal fluctuations, $\overline{\left\langle\delta T^{2}\right\rangle}$, close to the instability. The mean-square amplitude $\overline{\left\langle\delta T^{2}\right\rangle}$ of the temperature fluctuations can be related to the structure factor by Eq. (9), such that

$$
\begin{aligned}
\overline{\left\langle\delta T^{2}\right\rangle} & =\frac{1}{L} \int_{0}^{L} d z\left\langle\theta^{*}\left(\mathbf{x}_{\|}, z, t\right) \cdot \theta\left(\mathbf{x}_{\|}, z, t\right)\right\rangle \\
& =\frac{1}{\alpha^{2} \rho^{2}} \frac{1}{L} \int_{0}^{L} d z \int \frac{d^{2} \mathbf{q}}{(2 \pi)^{2}} S(q, z, z) .
\end{aligned}
$$

Due to the horizontal translational symmetry of the problem, this quantity does not depend on the point $\mathbf{x}_{\|}$in the horizontal plane at which is evaluated on the time $t$ at which is calculated. For the case of "slip-free" boundary conditions, substituting $z=z^{\prime}$ in Eq. (10) for $S\left(q, z, z^{\prime}\right)$ causes a problem 
associated with the short-range equilibrium contribution, $\delta\left(z-z^{\prime}\right)$; but since the quantity Eq. (22) that is experimentally observed actually depends on a double integral on $z$ and $z^{\prime}$, this problem is not relevant. Therefore, we can safely neglect the equilibrium contribution in the calculation of $\overline{\left\langle\delta T^{2}\right\rangle}$. Substituting Eq. (10) into Eq. (40), we thus obtain for the case of two free boundaries [29],

$$
\overline{\left\langle\delta T^{2}\right\rangle}=\frac{S_{E} \widetilde{S}_{\mathrm{NE}}^{0}}{\alpha^{2} \rho^{2} L(\sigma+1)} \frac{1}{2 \pi} \sum_{N=1}^{\infty} \int_{0}^{\infty} \frac{\tilde{q}^{2} q d q}{\left(\tilde{q}^{2}+N^{2} \pi^{2}\right)^{3}-R \tilde{q}^{2}} .
$$

The integral in Eq. (41) can be performed analytically, but the result is long and not particularly interesting. Asymptotically close to the convective instability we find

$$
\overline{\left\langle\delta T^{2}\right\rangle} \simeq \frac{S_{E}\left(\widetilde{S}_{\mathrm{NE}}^{0}\right)_{c}}{\alpha^{2} \rho^{2} L(\sigma+1)} \frac{1}{4 \pi^{2}} \frac{2 \sqrt{3}}{27 L^{2} \sqrt{-\epsilon}} .
$$

In Eq. (42), the symbol $\left(\widetilde{S}_{\mathrm{NE}}^{0}\right)_{c}$ means that the normalized amplitude of the nonequilibrium enhancement has to be evaluated at the critical temperature gradient. Using Eqs. (2) and (3), neglecting the contribution of the adiabatic temperature gradient, and using again the thermodynamic relation $\alpha^{2} D_{T}=[(\gamma-1) / \gamma] \lambda_{T} \varkappa_{T} / T$, we rewrite Eq. (42) as

$$
\overline{\left\langle\delta T^{2}\right\rangle} \simeq k_{B} T \frac{\left(\Delta T_{c}\right)^{2}}{\rho L \nu^{2}} \frac{\sqrt{3}}{54 \pi^{2}} \frac{\sigma^{2}}{\sigma+1} \frac{1}{\sqrt{-\epsilon}},
$$

where $\Delta T_{c}$ is the temperature difference which corresponds to the critical Rayleigh number. The behavior of the power of the thermal fluctuations, $\overline{\left\langle\delta T^{2}\right\rangle}$, has been studied by several authors $[39,41]$ in the framework of the standard SH model; asymptotically close to the instability this quantity is expressed as $[39,41]$

$$
\overline{\left\langle\delta T^{2}\right\rangle} \simeq k_{B} T \frac{\left(\Delta T_{c}\right)^{2}}{\rho L \nu^{2}} \frac{\sigma \tilde{q}_{c} \widetilde{c}^{2}}{2 \xi_{0} \tau_{0} R_{c}^{3}} \frac{1}{\sqrt{-\epsilon}},
$$

where $\tilde{c}=3 \tilde{q}_{c} \sqrt{R_{c}}, \xi_{0}$, and $\tau_{0}$ are dimensionless parameters appearing in the standard SH model. These parameters depend on the boundary conditions, and $\tau_{0}$ also depends on the Prandtl number. Numerical values of these parameters, for free and rigid boundaries, can be found in Table I of Hohenberg and Swift [26]. Substituting into Eq. (44) the values found in that table for free boundaries, we obtain exact numerical agreement with our current Eq. (43). We conclude that, for the case of free boundaries, our exact solution for $S(q)$, when evaluated close to the instability, shows perfect agreement with the standard SH model, not only for the dependence of the structure factor on $q$, but also for the magnitude of the amplitude of the nonequilibrium fluctuations.

For the case of rigid boundaries, we consider the divergence of $\overline{\left\langle\delta T^{2}\right\rangle}$ at the convective instability obtained in the first-order Galerkin approximation, Eq. (19). We first observe that, since the Galerkin approximation is a single-mode approximation, we do not encounter any problems associated with the short-range equilibrium part when substituting $z$ $=z^{\prime}$. We also note that the integration on $q$ of $\Lambda_{0}^{R}(q)$ is complicated, and since we are really interested only in the asymptotic behavior close to the instability, we prefer to use the SH approximant to the first-order Galerkin, which is easier to handle. Moreover, we have checked numerically that for a Prandtl number $\sigma=8$ and for $\epsilon$ within -0.01 from the instability, the difference between $\overline{\left\langle\delta T^{2}\right\rangle}$ calculated with the SH approximation and the same quantity calculated with the full Galerkin approximation, is less than $1 \%$. Thus, we substitute Eq. (39) into Eq. (40), taking into account that Eq. (39) is obtained through a double integration on $z$ and $z^{\prime}$ [see Eq. (22)] while Eq. (40) requires us to first substitute $z=z^{\prime}$ and then to average vertically. Evaluating the asymptotic behavior of the resulting integral close to the instability, we obtain

$$
\begin{aligned}
\overline{\left\langle\delta T^{2}\right\rangle}= & \frac{S_{E} \widetilde{S}_{\mathrm{NE}}^{0}}{\alpha^{2} \rho^{2} L} \frac{\widetilde{H}(\sigma)}{L^{2} R_{c}} \frac{6}{5} \frac{\pi}{\widetilde{\xi}_{0}^{2} \sqrt{-\epsilon}} \frac{1}{(2 \pi)^{2}} \\
& \times\left[\frac{\pi}{2}+\arctan \left(\frac{\widetilde{\xi}_{0}^{2} \tilde{q}_{c}^{2}}{\sqrt{-\epsilon}}\right)\right], \\
\simeq & k_{B} T \frac{\left(\Delta T_{c}\right)^{2}}{\rho L \nu^{2}} \frac{1}{4 \widetilde{\xi}_{0}^{2} R_{c}} \frac{\sigma^{2}}{(\sigma+0.515)} \frac{1}{\sqrt{-\epsilon}},
\end{aligned}
$$

where we have substituted the function $\widetilde{H}(\sigma)$ quoted in Table I for the case of rigid boundaries. Comparing Eq. (45) with the result obtained by substituting into Eq. (44) the parameters corresponding to rigid boundaries in the standard SH approximation (cf. Table I of Hohenberg and Swift [26]) we observe the following.

As in the case of free boundaries, we recover the divergence of $\overline{\left\langle\delta T^{2}\right\rangle}$ as $1 / \sqrt{-\epsilon}$, predicted by the standard $\mathrm{SH}$ model and confirmed experimentally by Wu et al. [39].

We also recover, in good approximation, the same kind of dependence of $\overline{\left\langle\delta T^{2}\right\rangle}$ on the Prandtl number: a factor $\sigma^{2} /(\sigma+0.515)$, to be compared with $\sigma^{2} /(\sigma+0.5117)$ predicted by the standard SH model.

The prefactor for the total power of the thermal noise calculated with our model, Eq. (45), for rigid boundaries is $3 \%$ smaller than the same quantity calculated with the standard SH model. This small difference shows that the Galerkin approximation proposed is this paper is a very good approximation indeed.

The experimental data presented by $\mathrm{Wu}$ et al. [39] were reanalyzed by Bodenschatz et al. [41] who concluded that the experimental results were consistent with the predictions of the SH model. Since our first-order Galerkin differs only $3 \%$ from the SH model, we conclude that our approximation yields also a satisfactory representation of these experimental results.

Many authors [23-26] have represented the $q$ dependence of the structure factor near the instability in terms of a Lorentzian profile centered at $\tilde{q}_{c}$ with a width proportional to $\epsilon$, and $\mathrm{Wu}$ et al. [39] have analyzed their experimental data in terms of such a Lorentzian profile. We remark that both 


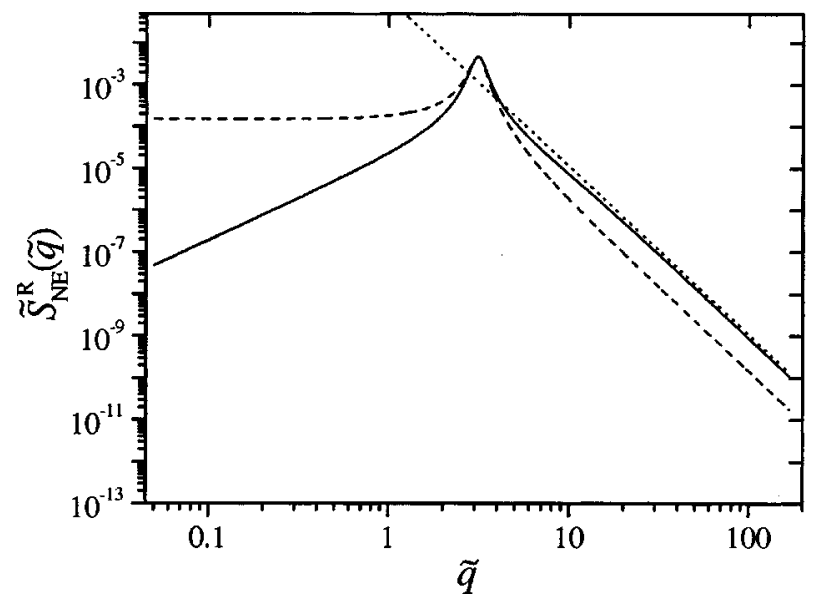

FIG. 4. Normalized nonequilibrium enhancement $\widetilde{S}_{\mathrm{NE}}^{R}(\tilde{q})$ of the structure factor of a fluid with $\sigma=8$, as a function of the dimensionless wave number near the convective instability $[\epsilon=(R$ $\left.\left.-R_{c}\right) / R_{c}=-0.01\right]$ for rigid boundaries. The solid curve represents the first-order Galerkin approximation given by Eq. (26). The dashed curve represents the corresponding SH approximation with the parameter values listed in the second row of Table I. The dotted line represents the exact expression for the nonequilibrium structure factor of the bulk fluid, which should be the correct asymptotic limit for large $\tilde{q}$.

the exact solution for free boundaries and the first-order Galerkin solution for rigid boundaries do not yield a Lorentzian profile close to the instability. Moreover, a Lorentzian does not recover the proper asymptotic behavior, implied by in Eqs. (27) -(30), for either small $q$ or large $q$. It even leads to an apparent divergence when one tries to calculate the power of the fluctuations by integrating $S(q)$ over all twodimensional wave vectors [26,39].

The SH approximation has been widely used in the literature to study the fluctuations close to the convective instability. We conclude this section by comparing the SH approximation with the Galerkin approximation derived in this paper. To do so, we have plotted in Fig. 4 the Galerkin approximation for the nonequilibrium enhancement $\widetilde{S}_{\mathrm{NE}}^{R}(\tilde{q})$ of the structure factor (solid curve), together with the corresponding SH approximation calculated with Eq. (39) and the parameter values listed in the second row of Table I (dashed curve). Both curves correspond to $\sigma=8$ and $\epsilon=-0.01$. For reference, we have also plotted in Fig. 4 the exact result for the bulk structure factor. A simple examination of this figure shows that, although $\mathrm{SH}$ represents the maximum of the structure factor quite well, it does not reproduce the proper asymptotic behaviors for large or small $\tilde{q}$. Although the $\mathrm{SH}$ approximation goes as $\tilde{q}^{-4}$ for large $q$, the prefactor multiplying this $\tilde{q}$ dependence is smaller than the correct value, which should be $(\sigma+1)^{-1}$. We note that the Galerkin approximation is, instead, only a few percent off. In the limit of small $q$, the $\mathrm{SH}$ approximation reaches a finite constant value, while the Galerkin approximation goes to zero as $q^{2}$. Recent measurements obtained by Oh and Ahlers [53] for the nonequilibrium structure factor of sulfur hexafluoride below the Rayleigh-Bénard instability turn out to be consistent with our theoretical prediction that $S(q)$ should exhibit a crossover from a $q^{-4}$ behavior for larger $q$ to a $q^{2}$ behavior for small $q$.

\section{CONCLUSIONS}

In this paper we have applied the Galerkin method so as to calculate the static structure factor of a nonequilibrium fluid from the linearized random Boussinesq equations, for both negative and positive values of the Rayleigh number, using "no-slip" boundary conditions. Explicit expressions for the nonequilibrium enhancement of the structure factor in the Galerkin approximation have been presented. The consequences for low-angle light-scattering and shadowgraph experiments have been elucidated. The resulting expression reproduces the $q^{-4}$ dependence of the nonequilibrium structure factor predicted theoretically [2-4] and confirmed experimentally [10-13] for negative Rayleigh numbers, it accounts for the saturation of the nonequilibrium enhancement of the intensity of the fluctuations at small wave numbers observed by Giglio and co-workers $[14,15,42,43]$ and it is consistent with the experimental observation by Ahlers and co-workers $[39,41,53]$ of the structure factor close to the convective instability. We have thus provided a unified approach for describing nonequilibrium fluctuations for both negative and positive values of the Rayleigh number, provided that $R<R_{c}$. A major conclusion is that fluctuating hydrodynamics with simple additive thermal noise is enough to account for the nonequilibrium structure factors measured experimentally. Hence, at least for the case of RayleighBénard convection, it does not appear necessary to look for "fancy" sources of noise, such as multiplicative, correlated, or colored noise [35,54].

The nonequilibrium structure factor obtained here for the case of two rigid boundaries exhibits qualitatively the same behavior as that deduced from an exact result derived for the case of two free boundaries in a previous publication [29]. The typical $q^{-4}$ divergence of the nonequilibrium structure factor crosses over to a $q^{2}$ dependence for extremely small scattering angles. Separating both behaviors there is a maximum in the scattered intensity, indicating that fluctuations with a particular wave vector are maximally enhanced. As the convective instability is approached the height of the maximum diverges. The wave number $\widetilde{q}_{\max }$ of the fluctuations that are maximally enhanced is close to the critical wave number $\tilde{q}_{c}$ for the convective instability and depends on the actual boundary conditions considered. Fluctuating patterns do appear in a fluid subjected to a stationary temperature gradient below the convective instability, even for negative Rayleigh numbers. To address the question how these fluctuating patterns below the Rayleigh-Bénard instability evolve into convection rolls above the instability requires a theoretical approach that goes beyond the linearized Boussinesq equations considered in the present paper [32].

\section{ACKNOWLEDGMENTS}

The authors are indebted to G. Ahlers and D. S. Cannell for some valuable comments. The research at the University 
of Maryland was supported by the Chemical Sciences, Geosciences and Biosciences Division of the Office of Basic Energy Sciences of the U.S. Department of Energy under Grant No. DE-FG-02-95ER14509. J.M.O.Z. acknowledges the hospitality of the Institute for Physical Science and Technology, University of Maryland, where part of the manuscript was prepared.

\section{APPENDIX: WAVE NUMBER OF MAXIMUM GROWTH RATE OF HYDRODYNAMIC PERTURBATIONS}

In Sec. VI we derived an expression for the wave number $\tilde{q}_{\max }$ corresponding to the maximum enhancement of the intensity of the nonequilibrium fluctuations, as a function of $\epsilon=\left(R-R_{c}\right) / R_{c}$ and of the Prandtl number $\sigma$. Another interesting quantity is the wave number $\tilde{q}_{m}$, corresponding to the maximum growth rate of perturbations around the quiescent conductive hydrodynamic state in the fluid layer $[30,33,46-$ 48]. While both $\tilde{q}_{\max }$ and $\tilde{q}_{m}$ approach $\tilde{q}_{c}$ as $\epsilon \rightarrow 0^{(-)}$, the two wave numbers differ for finite negative values of $\epsilon$ and this difference depends on the Prandtl number.

To illustrate the difference between $\tilde{q}_{\max }$, the wave number of maximum enhancement of fluctuations, and the wave number $\tilde{q}_{m}$, corresponding to the maximum growth rate, we first consider the case of two free boundaries, for which there exists a well-known analytic expression for $\tilde{g}\left(\tilde{q}_{\|}\right)$ $=g(\tilde{q} \|) \nu / L^{2}$, the dimensionless linear growth rate of the most unstable mode $[30,33]$

$$
\begin{aligned}
\tilde{g}\left(\tilde{q}_{\|}\right)= & -\frac{\left(\tilde{q}_{\|}^{2}+\pi^{2}\right)(\sigma+1)}{2 \sigma} \\
& \times\left\{1-\sqrt{1-\frac{4 \sigma}{(\sigma+1)^{2}}\left[1-\frac{\tilde{q}_{\|}^{2} R}{\left(\tilde{q}_{\|}^{2}+\pi^{2}\right)^{3}}\right]} .\right.
\end{aligned}
$$

Note that we are using the notation $g(\tilde{q} \|)$ for the linear growth rate, instead of the traditional $\sigma$ to avoid confusion with the Prandtl number. We observe in Eq. (A1) that the condition of marginal stability $\tilde{g}(\tilde{q} \|)=0$ is the same condition obtained in the main text from an analysis of the stochastic Boussinesq equations for the case of two free boundaries. Thus, to have the possibility of the linear growth rate to be zero, the Rayleigh number $R$ has to be larger than the critical Rayleigh number for free boundaries, $R_{c}=27 \pi^{4} / 4$. At $R=R_{c}$ the growth rate reaches the value zero at a single finite value of the wave number $\tilde{q}_{\|}=\tilde{q}_{c}=\pi / \sqrt{2}$. For $R$ $<R_{c}$, the maximum growth rate given by Eq. (A1) is always negative, independent of $\sigma$ or $\tilde{q} \|$; this means that the conductive solution is stable. For $R>R_{c}$ there are values of $q_{\|}$ for which the corresponding growth rate is positive, indicating that an instability develops in the system. A plot of Eq. (A1) shows that for $R \leq R_{c}$ there is a maximum of the growth rate for a particular value $\tilde{q}_{\|}=\tilde{q}_{m}$. At $R=R_{c}$, the maximum is located at $\tilde{q}_{c}$ and the value of the growth rate at the maxi- mum is zero. From Eq. (A1) one readily deduces an analytical expression for the position of the maximum at $R \leq R_{c}$,

$$
\tilde{q}_{m}=\tilde{q}_{c}\left[1+\frac{1}{4} \epsilon+\frac{3 \sigma^{2}+2 \sigma+3}{16(\sigma+1)^{2}} \epsilon^{2}+O\left(\epsilon^{3}\right)\right] .
$$

On comparing Eq. (A2) with Eq. (33), we already observe quite clearly the difference between the wave number of maximum growth rate of hydrodynamic perturbations, $\tilde{q}_{m}$, and the wave number, $\tilde{q}_{\max }$ of fluctuations maximally enhanced. From Eq. (33) we see that $\tilde{q}_{\max }$ does not contain the term linear in $\epsilon$ in the case of free boundaries.

For the case of rigid boundaries, the maximum growth rate cannot be calculated analytically, but has been evaluated numerically by Domínguez-Lerma et al. [48]. However, it is possible to calculate analytically a Galerkin approximation to the linear growth rate, much in the spirit of the calculations presented in the main text of the paper. To perform this approximate analytical calculation we consider the deterministic Boussinesq equations (thus $\delta \mathrm{T}=\delta \mathbf{Q}=0$ ), Fourier transformed in the horizontal plane, but not in time, so that from Eqs. (5) in the main text we obtain

$$
\frac{\partial}{\partial t}\left[\mathcal{D} w\left(t, q_{\|}, z\right)\right]=\nu\left[\mathcal{D}^{2} w\left(t, q_{\|}, z\right)\right]-q_{\|}^{2} \alpha g \theta\left(t, q_{\|}, z\right),
$$

$$
\frac{\partial}{\partial t} \theta\left(t, q_{\|}, z\right)=D_{T}\left[\mathcal{D} \theta\left(t, q_{\|}, z\right)\right]-\nabla T_{0} w\left(t, q_{\|}, z\right),
$$

where the differential operator $\mathcal{D}$ was defined in Eq. (8). Now we look for approximate solutions to the deterministic Eqs. (A3) whose dependence on the vertical coordinate $z$ is expressed in terms of the same Galerkin polynomials used in Sec. III for the solution of the stochastic Boussinesq equations. Thus we consider perturbations of the form

$$
\begin{gathered}
w\left(t, \mathbf{q}_{\|}, z\right)=\exp \left[g\left(q_{\|}\right) t\right] w_{0}\left(q_{\|}\right)\left(\frac{z}{L}-\frac{z^{2}}{L^{2}}\right)^{2}, \\
\theta\left(t, q_{\|}, z\right)=\exp \left[g\left(q_{\|}\right) t\right] \theta_{0}\left(q_{\|}\right)\left(\frac{z}{L}-\frac{z^{2}}{L^{2}}\right),
\end{gathered}
$$

which, evidently, do fulfill the no-slip boundary conditions, Eqs. (12). To calculate an approximate solution to Eq. (A3) of the form given by Eq. (A4), we substitute Eq. (A4) into Eq. (A3), and project the first resulting equation onto the first Galerkin polynomial and the second equation onto the second Galerkin polynomial. After switching to dimensionless variables, we obtain the following set of linear algebraic equations for the dimensionless amplitudes $\tilde{w}_{0}\left(\tilde{q}_{\|}\right)$ $=\left(L / D_{T}\right) w_{0}\left(q_{\|}\right)$and $\widetilde{\theta}_{0}\left(\tilde{q}_{\|}\right)=\left(\alpha g L^{3} / \nu D_{T}\right) \theta_{0}\left(q_{\|}\right)$: 


$$
\left(\begin{array}{cc}
\frac{\tilde{g}\left(\tilde{q}_{\|}\right)\left(\tilde{q}_{\|}^{2}+12\right)}{630}+\frac{\left(\tilde{q}_{\|}^{4}+24 \tilde{q}_{\|}^{2}+504\right)}{630} & -\frac{\tilde{q}_{\|}^{2}}{140} \\
-\frac{R}{140 \sigma} & \frac{\tilde{g}\left(\tilde{q}_{\|}\right)}{30}+\frac{\left(\tilde{q}_{\|}^{2}+10\right)}{30 \sigma}
\end{array}\right)\left(\begin{array}{c}
\tilde{w}_{0}\left(\tilde{q}_{\|}\right) \\
\tilde{\theta}_{0}\left(\tilde{q}_{\|}\right)
\end{array}\right)=0,
$$

where the dimensionless growth rate is again $\tilde{g}=g \nu / L^{2}$. To have a solution of Eq. (A5) different from zero, the determinant of the matrix has to be zero. From the corresponding secular equation, we solve for the linear growth rate $\tilde{g}$, obtaining

$$
\tilde{g}(\tilde{q} \|)=-\frac{1}{2} \frac{\left(\tilde{q}_{\|}^{2}+10\right)\left[\sigma+\widetilde{A}\left(\tilde{q}_{\|}\right)\right]}{\sigma \widetilde{A}\left(\tilde{q}_{\|}\right)}\left\{1-\sqrt{1-\frac{\sigma \widetilde{A}\left(\tilde{q}_{\|}\right)}{7\left[\sigma+\widetilde{A}\left(\tilde{q}_{\|}\right)\right]^{2}}\left[28-\frac{27 \tilde{q}_{\|}^{2} R}{\left(\widetilde{q}_{\|}^{2}+10\right)\left(\tilde{q}_{\|}^{4}+24 \tilde{q}_{\|}^{2}+504\right)}\right]},\right.
$$

where the function $\widetilde{A}(\widetilde{q} \|)$ is given by

$$
\widetilde{A}\left(\tilde{q}_{\|}\right)=\frac{\left(\tilde{q}_{\|}^{2}+10\right)\left(\tilde{q}_{\|}^{2}+12\right)}{\left(\tilde{q}_{\|}^{4}+24 \widetilde{q}_{\|}^{2}+504\right)} .
$$

Actually there are two solutions for the growth rate $\tilde{g}(\tilde{q} \|)$; in Eq. (A6) we have displayed the larger one. We observe from Eq. (A6) that the condition of marginal stability $\tilde{g}(\tilde{q} \|)=0$ is the same condition, Eq. (21), obtained in the main text from an analysis of the stochastic Boussinesq equations in the first-order Galerkin approximation. We recall that for Eq. (21) to hold, the Rayleigh number $R$ has to be larger than a critical Rayleigh number $R_{c} \simeq 1708$, which is the first-order Galerkin approximation to the critical Rayleigh number for rigid boundaries. At $R=R_{c}$ the growth rate reaches the value zero at a single finite value of the wave number, $\tilde{q}_{\|}=\tilde{q}_{c}$ $\simeq 3.1163$. For $R<R_{c}$, the maximum growth rate, given by Eq. (A6), is always negative, independent of $\sigma$ or $\tilde{q}_{\|}$, meaning that the conductive solution is stable. For $R>R_{c}$ there are values of $q_{\|}$for which the corresponding growth rate $\tilde{g}(\tilde{q} \|)$ is positive, so that the conductive solution becomes unstable.

A plot of Eq. (A6) is qualitatively very similar to a plot of Eq. (A1) for the case of free boundaries; it shows that for $R \leqq R_{c}$ there is a maximum of the growth rate $\tilde{g}\left(\tilde{q}_{\|}\right)$for a particular value $\tilde{q}_{\|}=\tilde{q}_{m}$. At $R=R_{c}$, the maximum is located at $\tilde{q}_{c}$ and the value of the growth rate at the maximum is zero. Taking the derivative of $\tilde{g}\left(\tilde{q}_{\|}\right)$with respect to $\tilde{q}_{\|}$, we can deduce form Eq. (A6) an analytical expression for the position of the maximum at $R \leq R_{c}$. Specifically, we obtain

$$
\tilde{q}_{m}=\tilde{q}_{c}\left[1+\alpha(\sigma) \epsilon+O\left(\epsilon^{2}\right)\right],
$$

where some long algebraic calculations yield

$$
\begin{aligned}
\alpha(\sigma)= & \frac{35\left(\tilde{q}_{c}^{2}+12\right)^{4}\left(\tilde{q}_{c}^{2}+10\right)^{2}}{12 \tilde{q}_{c}^{6}\left(61987 \tilde{q}_{c}^{4}+165730 \tilde{q}_{c}^{2}+16132820\right)} \\
& \times \frac{21\left(\tilde{q}_{c}^{2}+12\right) \sigma+5\left(\tilde{q}_{c}^{2}-4\right)}{\tilde{q}_{c}^{2} \sigma+5} \\
\simeq & \frac{0.339(\sigma+0.063)}{\sigma+0.515} .
\end{aligned}
$$

The wave number corresponding to the maximum of the linear growth rate has been investigated numerically by Domínguez-Lerma et al. [48] for the case of rigid boundaries, who have proposed for the coefficient $\alpha(\sigma)$ in Eq. (A8) the empirical equation

$$
\alpha(\sigma)=0.0494+\frac{0.295 \sigma}{\sigma+0.509} .
$$

In Fig. 5 we have plotted $\alpha(\sigma)$ as a function of the Prandtl

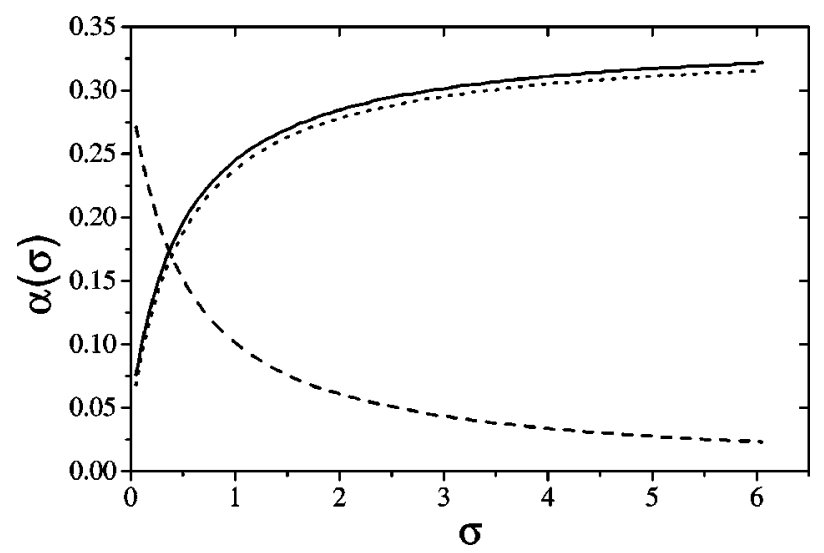

FIG. 5. Values of the linear coefficient $\alpha(\sigma)$ in the expansion, Eq. (A8), for the maximum wave number $\tilde{q}_{m}$ of the hydrodynamic perturbations. Solid curve, obtained analytically from a Galerkin approximation [Eq. (A9)]. Dotted curve, calculated numerically by Domínguez-Lerma et al. [48]. Dashed curve, linear coefficient in the expansion, Eq. (34), for the wave number $\tilde{q}_{\max }$ of maximum enhancement of fluctuations. 
number. The solid curve represents Eq. (A9), obtained here analytically using a Galerkin approximation and the dotted curve represents the empirical relationship, Eq. (A10), proposed by Domínguez-Lerma et al. [48]. A simple inspection of Fig. 5 shows that the Galerkin method provides a very good approximation for the wave number of the maximum growth rate of hydrodynamic perturbations; for the range of Prandtl numbers displayed in the figure, the difference between the value of $\alpha(\sigma)$ from our Galerkin approximation and from the numerical results of Domínguez-Lerma et al. is less than $2 \%$.

We can compare Eqs. (A8)-(A10) for the wave number $q_{m}$ of maximum linear growth rate of hydrodynamic perturbations with Eq. (34) in the main text for the wave number $q_{\max }$ of fluctuations maximally enhanced. We observe that, although at $R=R_{c}$ both maxima are located at $\tilde{q}_{c}$, for $R$ $\lessgtr R_{c}$ significant differences do appear between their positions. To show these difference we have added in Fig. 5, as a dashed curve, the value of $\alpha(\sigma)$ deduced from Eq. (34) for the wave number $q_{\max }$ of fluctuations maximally enhanced. We observe in Fig. 5 that the difference is important and it increases with the Prandtl number. As commented before, this difference is mathematically due to the fact that the wave number $q_{m}$ of maximum growth rate is obtained from solving the deterministic Boussinesq equations, while the wave number $q_{\max }$ of fluctuations maximally enhanced is obtained from solving the stochastic Boussinesq equations. We conclude that the quantity experimentally accessible for shadowgraph of light scattering below the instability, is the wave number $\tilde{q}_{\max }$ of fluctuations maximally enhanced and not $\tilde{q}_{m}$.
[1] J. R. Dorfman, T. R. Kirkpatrick, and J. V. Sengers, Annu. Rev. Phys. Chem. 45, 215 (1994).

[2] T. R. Kirkpatrick, E. G. D. Cohen, and J. R. Dorfman, Phys. Rev. A 26, 995 (1982).

[3] D. Ronis and I. Procaccia, Phys. Rev. A 26, 1812 (1982).

[4] R. Schmitz and E. G. D. Cohen, J. Stat. Phys. 39, 285 (1985).

[5] R. Schmitz, Phys. Rep. 171, 1 (1988).

[6] B. M. Law and J. V. Sengers, J. Stat. Phys. 57, 531 (1989).

[7] P. N. Segrè and J. V. Sengers, Physica A 198, 46 (1993).

[8] P. N. Segrè, R. Schmitz, and J. V. Sengers, Physica A 195, 31 (1993).

[9] B. J. Berne and R. Pecora, Dynamic Light Scattering (Wiley, New York, 1976).

[10] B. M. Law, P. N. Segrè, R. W. Gammon, and J. V. Sengers, Phys. Rev. A 41, 816 (1990).

[11] P. N. Segrè, R. W. Gammon, J. V. Sengers, and B. M. Law, Phys. Rev. A 45, 714 (1992).

[12] W. B. Li, P. N. Segrè, R. W. Gammon, and J. V. Sengers, Physica A 204, 399 (1994).

[13] W. B. Li, K. J. Zhang, J. V. Sengers, R. W. Gammon, and J. M. Ortiz de Zárate, Phys. Rev. Lett. 81, 5580 (1998).

[14] A. Vailati and M. Giglio, Phys. Rev. Lett. 77, 1484 (1996).

[15] A. Vailati and M. Giglio, Prog. Colloid Polym. Sci. 104, 76 (1997).

[16] A. S. Tremblay, M. Arai, and E. D. Siggia, Phys. Rev. A 23, 1451 (1981).

[17] L. S. García-Colín and R. M. Velasco, Phys. Rev. A 26, 2187 (1982).

[18] R. Schmitz and E. G. D. Cohen, Phys. Rev. A 35, 2602 (1987).

[19] D. Beysens, Y. Gabarros, and G. Zalczer, Phys. Rev. Lett. 45, 403 (1980).

[20] H. Kiefte, M. J. Clouter, and R. Penney, Phys. Rev. B 30, 4017 (1984).

[21] R. N. Suave and A. R. B. de Castro, Phys. Rev. B 53, 5330 (1996).

[22] J. B. Swift and P. C. Hohenberg, Phys. Rev. A 15, 319 (1977).

[23] T. R. Kirkpatrick and E. G. D. Cohen, J. Stat. Phys. 33, 639 (1983).

[24] R. Schmitz and E. G. D. Cohen, J. Stat. Phys. 40, 431 (1985).
[25] H. van Beijeren and E. G. D. Cohen, J. Stat. Phys. 53, 77 (1988).

[26] P. C. Hohenberg and J. B. Swift, Phys. Rev. A 46, 4773 (1992).

[27] J. M. Ortiz de Zárate, R. Pérez Cordón, and J. V. Sengers, Physica A 291, 113 (2001).

[28] J. M. Ortiz de Zárate and L. Muñoz Redondo, Eur. Phys. J. B 21, 135 (2001).

[29] J. M. Ortiz de Zárate and J. V. Sengers, Physica A 300, 25 (2001).

[30] S. Chandrasekhar, Hydrodynamic and Hydromagnetic Stability (Oxford University Press, Oxford, 1961).

[31] V. Zaitsev and M. Shliomis, Zh. Eksp. Teor. Fiz. 59, 1583 (1970) [Sov. Phys. JETP 32, 866 (1971)].

[32] M. C. Cross and P. C. Hohenberg, Rev. Mod. Phys. 65, 851 (1993).

[33] P. Manneville, Dissipative Structures and Weak Turbulence (Academic Press, San Diego, 1990).

[34] D. J. Tritton, Physical Fluid Dynamics, 2nd ed. (Oxford University Press, Oxford, 1988).

[35] J. García Ojalvo and J. M. Sancho, Noise in Spatially Extended Systems (Springer, New York, 1999).

[36] K. Staliunis, Phys. Rev. E 64, 066129 (2001).

[37] J. V. Sengers and J. M. Ortiz de Zárate, in Thermal Nonequilibrium Phenomena in Fluid Mixtures, edited by W. Köhler and S. Wiegand (Springer, Berlin, 2002), p. 121.

[38] J. V. Sengers and J. M. Ortiz de Zárate, Rev. Mex. Fis. (to be published).

[39] M. Wu, G. Ahlers, and D. S. Cannell, Phys. Rev. Lett. 75, 1743 (1995).

[40] J. R. deBruyn, E. Bodenschatz, S. W. Morris, S. P. Trainoff, Y. Hu, D. S. Cannell, and G. Ahlers, Rev. Sci. Instrum. 67, 2043 (1996).

[41] E. Bodenschatz, W. Pesch, and G. Ahlers, Annu. Rev. Fluid Mech. 32, 709 (2000).

[42] A. Vailati and M. Giglio, Nature (London) 390, 262 (1997).

[43] D. Brogioli and A. Vailati, and M. Giglio, Phys. Rev. E 61, R1 (2000).

[44] S. P. Trainoff and D. S. Cannell, Phys. Fluids 14, 1340 (2002). 
[45] D. Brogioli, A. Vailati, and M. Giglio, J. Phys.: Condens. Matter 12, A39 (2000).

[46] J. Westried, Y. Pomeau, M. Dubois, C. Normand, and P. Bergae, J. Phys. (Paris) 39, 725 (1978).

[47] M. C. Cross, Phys. Fluids 23, 1727 (1980).

[48] M. A. Domínguez-Lerma, G. Ahlers, and D. S. Cannell, Phys. Fluids 27, 856 (1984).

[49] C. Normand, Y. Pomeau, and M. G. Velarde, Rev. Mod. Phys.
49, 581 (1977).

[50] R. Graham, Phys. Rev. A 10, 1762 (1974).

[51] R. Graham and H. Pleiner, Phys. Fluids 18, 130 (1975).

[52] M. A. Scherer, G. Ahlers, F. Hörner, and I. Rehberg, Phys. Rev. Lett. 85, 3754 (2000).

[53] J. Oh and G. Ahlers (private communication).

[54] J. García Ojalvo, A. Hernández Machado, and J. M. Sancho, Phys. Rev. Lett. 71, 1542 (1993). 